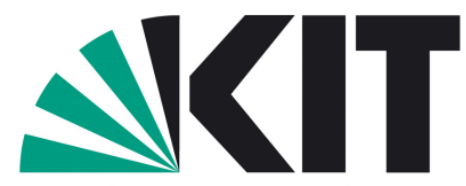

Karlsruher Institut für Technologie

\title{
Model-Aware Newton-Type Inversion Scheme For Electrical Impedance Tomography
}

\author{
R. Winkler \\ A. Rieder \\ Preprint 14/04
}

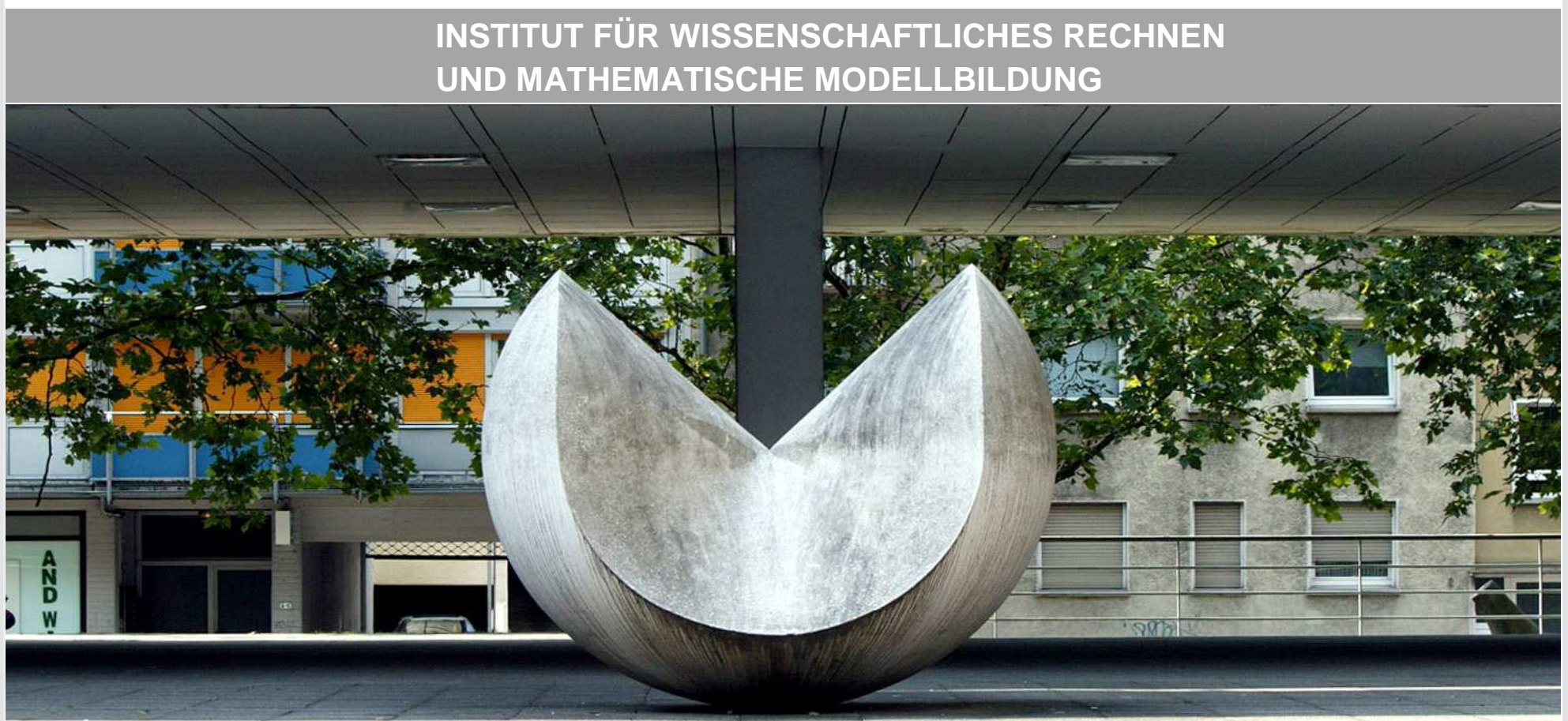

KIT - Universität des Landes Baden-Wurttemberg und

nationales Forschungszentrum in der Helmholtz-Gemeinschaft 


\section{Anschriften der Verfasser:}

Dipl.-Math. techn. Robert Winkler

Institut für Angewandte und Numerische Mathematik

Karlsruher Institut für Technologie (KIT)

D-76128 Karlsruhe

Prof. Dr. Andreas Rieder

Institut für Angewandte und Numerische Mathematik

Karlsruher Institut für Technologie (KIT)

D-76128 Karlsruhe 


\title{
MODEL-AWARE NEWTON-TYPE INVERSION SCHEME FOR ELECTRICAL IMPEDANCE TOMOGRAPHY
}

\author{
ROBERT WINKLER AND ANDREAS RIEDER
}

\begin{abstract}
Electrical impedance tomography is a non-invasive method for imaging the electrical conductivity of an object from voltage measurements on its surface. This inverse problem suffers in three respects: It is highly nonlinear, severely ill-posed and highly under-determined. To obtain yet reasonable reconstructions, maximal information needs to be gathered from the model and extracted from the data in all stages of the reconstruction procedure. We will present a holistic reconstruction framework which estimates the unknown model-specific parameters, i.e. background conductivity, contact impedance, and noise level, before solving the full nonlinear problem with a Newton-type method. Therein, a novel conductivity transformation decreases nonlinearity while a weighting scheme resolves the under-determinedness by promoting the reconstruction of piecewise constant conductivities. This way we increase robustness, speed, and reconstruction accuracy. Moreover, our method is easy to use and applies to a wide range of settings as it is free of design parameters. Being an absolute imaging method, no measured calibration data is required. We demonstrate the performance of this concept numerically for simulated and measured data.
\end{abstract}

\section{BACKGROUND}

Electrical impedance tomography (EIT) is a non-invasive imaging method with applications in clinical diagnostics, patient monitoring, and process tomography. Its purpose is to determine the spacial conductivity distribution of an object from measurements on its boundary. To that end, electrical currents are applied through electrodes attached to the object surface and the resulting potential distributions are measured on the same electrodes. An accurate mathematical description of this process is given by the complete electrode model (CEM, [19]), which incorporates the shunting effect of the electrodes and a contact impedance occurring at the electrode-object interface.

The mathematical task of EIT is the inverse conductivity problem (ICP), i.e. to determine the conductivity (or some information about it) from the current-potential measurement pairsa highly nonlinear, under-determined and unstable inverse problem. Many popular solution methods use iterated linearizations of the forward operator (Newton-type methods). In addition to the measurements, the electrode contact impedance and a good initial guess of the conductivity are required as Newton's method converges only locally in general. Moreover, error related regularization parameters need to be specified, like the smoothing parameter for Tikhonov regularization, the noise level for Morozov's discrepancy principle or the parameter means and covariances in the Bayesian framework.

Aim and structure of this work. Generic Newton-type approaches assume the model parameters to be known, or chosen manually, to obtain meaningful reconstructions. While the object and electrode geometry can be accessed from "outside" or by other imaging modalities, parameters like the contact impedances, the background-conductivity and the noise are difficult to obtain and highly problem-specific.

Date: December 9, 2014.

2010 Mathematics Subject Classification. Primary 65N20; Secondary 65F22, 92C55.

The work of the authors was supported by the German Research Foundation (DFG) under grant RI 975/7-1. 
To overcome these difficulties, we suggest a systematic way to estimate the contact impedances and the background conductivity using Green's identity for the CEM in section 3.1. Moreover, we use a symmetry property of the model to estimate the measurement error in section 3.2 .

In section 3.3, we investigate the nonlinearity and constrainedness of the homogeneous conductivity problem, i.e. of determining a constant conductivity by linearization, and consider various transformations (e.g. the popular log-conductivity [2, 9]) to reduce nonlinearity and to obtain an unconstrained problem.

In section 3.4, we resolve the under-determinedness of the discretized linearized problem by introducing a reconstruction prior which encourages the reconstruction of piecewise constant conductivities whenever conductivity coefficient updates are indistinguishable in the linearization. This prior is incorporated as a weighting scheme for the regularized conjugate gradient iteration REGINN [16] to compute the Newton updates in section 3.5 .

All model-specific additions to a generic Newton-type inversion scheme are summarized in a model-aware Newton-type inversion scheme (MANTIS) in section 3.6.

Finally, the performance of this scheme is demonstrated for noisy simulated data and measured tank data in section 4 ,

All following considerations are independent of the spatial dimension. We formulate them in a two-dimensional setting and present numerical computations in a 2D model, but MANTIS can readily be applied to three dimensional settings.

\section{Preliminaries}

A potential $u \in H^{1}(\Omega)$ on a source-free, simply connected domain $\Omega \subset \mathbb{R}^{2}$ with piecewise Lipschitz boundary satisfies the conservation of charge

$$
\nabla \cdot \sigma \nabla u=0 \text { on } \Omega,
$$

where $\sigma \in L_{+}^{\infty}(\Omega)=\left\{\varphi \in L^{\infty}(\Omega) \mid \varphi \geq c>0\right.$ a.e. for some $\left.c \in \mathbb{R}_{>0}\right\}$ is the isotropic conductivity coefficient.

2.1. Forward Model. A potential is uniquely defined by its boundary trace $f=\left.u\right|_{\partial \Omega}$ or, up to a constant, by its outer normal boundary current $i_{\nu}=\sigma \partial u / \partial \nu$. A pair

$$
\left(f, i_{\nu}\right) \in H_{\diamond}^{-1 / 2}(\partial \Omega) \times H_{\diamond}^{1 / 2}(\partial \Omega), \quad \text { where } \quad H_{\diamond}:=\left\{\varphi \in H \mid\langle\varphi, 1\rangle_{H, H^{*}}=0\right\},
$$

is called (continuum) Neumann-Dirichlet (ND) datum. The linear ND operator

$$
H_{\diamond}^{-1 / 2}(\partial \Omega) \rightarrow H_{\diamond}^{1 / 2}(\partial \Omega), \quad i_{\nu} \mapsto f,
$$

is well-defined and one-to-one. The map

$$
\mathcal{F}: L_{+}^{\infty}(\Omega) \rightarrow \mathcal{L}\left(H_{\diamond}^{-1 / 2}(\partial \Omega), H_{\diamond}^{1 / 2}(\partial \Omega)\right), \quad \sigma \mapsto\left(i_{\nu} \mapsto f\right),
$$

is called the forward operator of the continuum model; see [1].

In practice, net current patterns $I \in \mathbb{R}_{\diamond}^{L}$ are applied and potential vectors $U \in \mathbb{R}_{\diamond}^{L}$ are measured via simply connected, separated electrodes at the object surface

$$
E_{1}, \ldots, E_{L} \subset \partial \Omega, \quad L \in \mathbb{N}_{\geq 2} .
$$

Each electrode-domain interface has a contact impedance $z_{l}>0$ causing a potential drop $z_{l} i_{\nu}$, $l=1, \ldots, L$. The interior and boundary potential $(u, U) \in H_{\diamond}^{1}(\Omega) \times \mathbb{R}_{\diamond}^{L}$ for an applied current $I \in \mathbb{R}_{\diamond}^{L}$ are given by the CEM as the unique solution of

$$
a((u, U),(w, W))=\sum_{l=1}^{L} I_{l} W_{l} \quad \text { for all }(w, W) \in H^{1}(\Omega) \times \mathbb{R}_{\diamond}^{L},
$$


where $a:\left(H^{1}(\Omega) \times \mathbb{R}_{\diamond}^{L}\right) \times\left(H^{1}(\Omega) \times \mathbb{R}_{\diamond}^{L}\right) \rightarrow \mathbb{R}$,

$$
a((v, W),(w, W))=\int_{\Omega} \sigma \nabla v \cdot \nabla w \mathrm{~d} x+\sum_{l=1}^{L} \frac{1}{z_{l}} \int_{E_{l}}\left(v-V_{l}\right)\left(w-W_{l}\right) \mathrm{d} S .
$$

For details, see [19]. The ND (or current-to-voltage) map of the CEM is

$$
\mathbb{R}_{\diamond}^{L} \rightarrow \mathbb{R}_{\diamond}^{L}, \quad I \mapsto U
$$

and the forward operator of the CEM is

$$
F: L_{+}^{\infty}(\Omega) \supset \mathcal{D}(F) \rightarrow \mathcal{L}\left(\mathbb{R}_{\diamond}^{L}\right), \quad \sigma \mapsto(I \mapsto U) .
$$

Note that $F(\sigma) \in \mathcal{L}\left(\mathbb{R}_{\diamond}^{L}\right)$ is symmetric, thus $\operatorname{dim}(\operatorname{Range}(F)) \leq \frac{L(L-1)}{2}$. In practice, the data is given in a certain measurement basis of $M \in \mathbb{N}$ measurements; that is, we know a set of current vectors $\mathcal{I}=\left(I^{(1)}, \ldots, I^{(M)}\right) \in\left(\mathbb{R}_{\diamond}^{L}\right)^{M}$ and their corresponding potentials $\mathcal{U}=\left(U^{(1)}, \ldots, U^{(M)}\right) \in$ $\left(\mathbb{R}_{\diamond}^{L}\right)^{M}$ satisfying $F(\sigma) \mathcal{I}=\mathcal{U}$.

Moreover, $F$ is Fréchet differentiable. The Fréchet derivative at conductivity $\sigma$ in direction $\eta \in L^{\infty}(\Omega)$ is given as

$$
F^{\prime}(\sigma)[\eta] I=U^{\prime}
$$

where $U^{\prime}$ is the second component of $\left(u^{\prime}, U^{\prime}\right)$ solving

$$
a\left(\left(u^{\prime}, U^{\prime}\right),(w, W)\right)=-\int_{\Omega} \eta \nabla u \nabla w \mathrm{~d} x \quad \text { for all } \quad(w, W) \in H^{1}(\Omega) \times \mathbb{R}_{\diamond}^{L},
$$

and $(u, U)$ is the solution of $(2.3)$ for conductivity $\sigma$ and current $I$; see [11, 12]. In particular, the Fréchet derivative entries can be sampled by

$$
\left(F^{\prime}(\sigma)[\eta]\right)_{l, m}=-\int_{\Omega} \eta \nabla u^{(l)} \nabla u^{(m)} \mathrm{d} x, \quad l, m=1, \ldots, L .
$$

Here, $u^{(l)}$ is the potential corresponding to the $l$ th "virtual" unit current $I^{(l)} \in \mathbb{R}^{L}, I_{k}^{(l)}=\delta_{l k}$; see [15, 10] for details.

2.2. Inverse Model. The ICP for the CEM is to find a (regularized) solution to the nonlinear system

$$
F(\sigma) \mathcal{I}=\mathcal{U}, \quad \sigma \in \mathcal{D}(F) .
$$

To that end, the conductivity space is usually restricted to piecewise constant functions on a partition $\mathcal{P}=\left\{\Omega_{1}, \ldots, \Omega_{P}\right\}, P \in \mathbb{N}$, of $\Omega$. For $\mathcal{D}_{\mathcal{P}}=\operatorname{span}\left\{\chi_{\Omega_{1}}, \ldots, \chi_{\Omega_{P}}\right\}$, we define

$$
\mathcal{D}(F):=\mathcal{D}_{\mathcal{P}+}:=\mathcal{D}_{\mathcal{P}} \cap L_{+}^{\infty}(\Omega), \quad \mathcal{D}_{\mathcal{P}+} \supset \sigma \widehat{=} \boldsymbol{\sigma}=\left(\sigma_{1}, \ldots, \sigma_{P}\right)^{\top} \in \mathbb{R}_{+}^{P},
$$

where we identify ${ }^{1}$ a conductivity $\sigma$ with its coefficient vector $\boldsymbol{\sigma}$. Often, $\mathcal{P}$ is chosen as a finite element triangulation of $\Omega$, although other, more problem-specific discretizations have been suggested [22, 4, 13, 3]. Note that even in this finite-dimensional setting, 2.5) is usually underdetermined, that is $P>\frac{L(L-1)}{2}$. Denoting by $\operatorname{col}(\cdot)$ the operation of stacking a matrix into a column vector column-wise, we define the sensitivity matrix $\mathcal{S} \in \mathbb{R}^{L M \times P}$ column-wise by

$$
(\mathcal{S})_{p}=\operatorname{col}\left(F^{\prime}(\sigma)\left[\chi_{\Omega_{p}}\right] \mathcal{I}\right) \quad \text { for } \quad p=1, \ldots, P .
$$

Discretization of the inverse problem. As the forward operator $F$ is Fréchet differentiable, regularized Newton-type methods are a natural choice for solving (2.5) numerically; that is, a locally linearized problem is solved in each iteration to solve the nonlinear problem. A basic scheme is shown in Algorithm 1. The evaluation of the forward operator and its Fréchet derivative in each iteration can be obtained using the finite element method (FEM); see [15].

\footnotetext{
${ }^{1}$ Coefficient vectors in $\mathbb{R}_{+}^{P}$ and $\mathbb{R}^{P}$ are denoted by the boldface symbols of their continuum correspondences in $\mathcal{D}_{\mathcal{P}+}$ and $\mathcal{D}_{\mathcal{P}}$, respectively, throughout this work.
} 


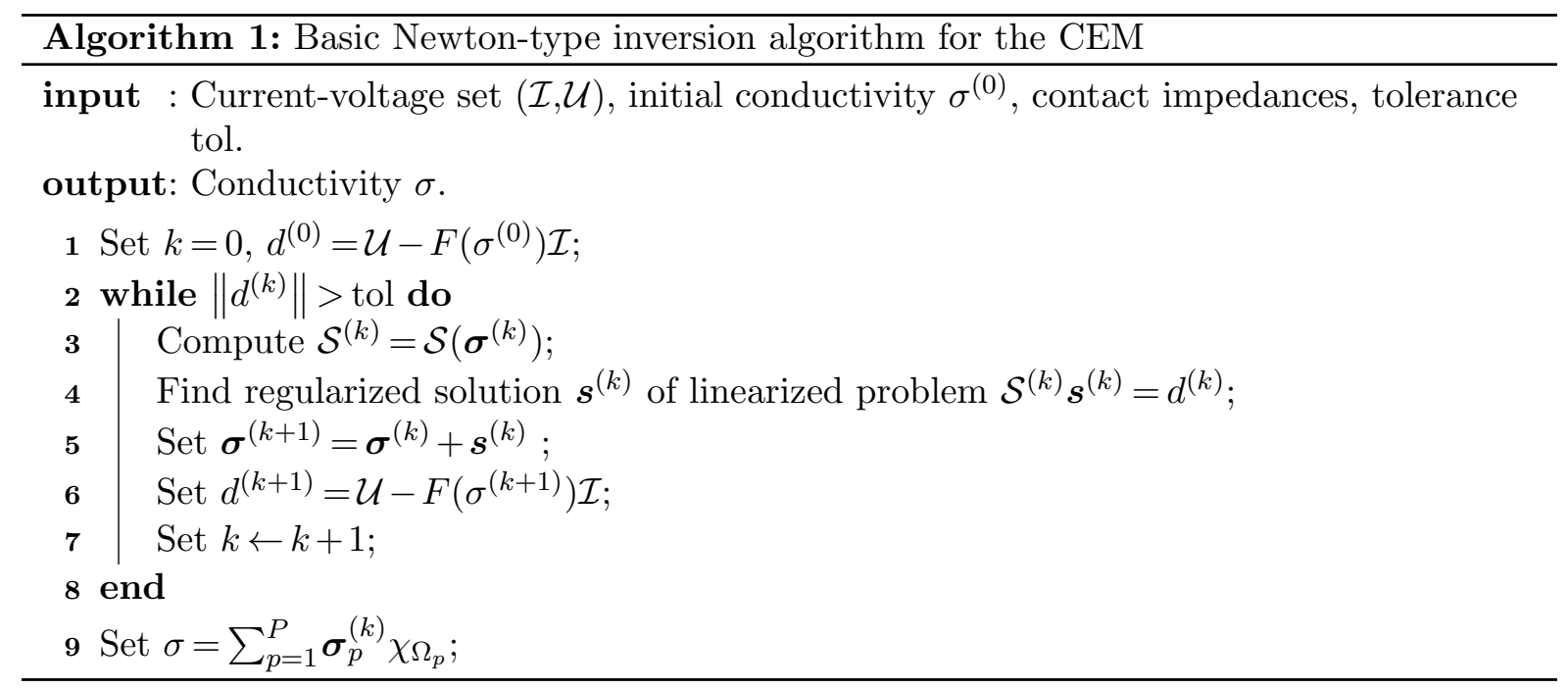

\section{Model-SPECIFIC MOdifiCATIONS FOR NEWTON-TYPE ALGORITHMS}

Our goal is to incorporate a set of modifications and extensions to Algorithm 1 in light of available CEM model information for a given setting. To that end, we proceed in several steps: First, we introduce a "best constant" conductivity and contact impedance estimate and determine a tolerance level by estimating the data noise level from the measurements (Algorithm 1 initialization). Next, we suggest a transformation of the forward problem to solve an equivalent problem with better linearization and constraint properties (lines 3 5). Finally, we present a sensitivity-based weighting scheme for each Newton-step which resolves the underdeterminedness of the linearized problem (line 4). The resulting Newton update is independent of the underlying conductivity discretization and reflects the prior assumption of a piecewise constant conductivity.

3.1. Conductivity and contact impedance initialization. As Newton-type algorithms only convergence locally in general, a proper initial guess is crucial. Most commonly, the conductivity is initialized to a known - or guessed - background conductivity manually, or it is estimated from the data as a best matching constant.

Continuum model approximation. A well-known [4] initial guess of the conductivity is based on the continuum model, more precisely on the fact that (2.1) is Laplace's equation for constant conductivities. Determining the constant can be transformed to a linear problem: The constant conductivity to ND operator

$$
\mathbb{R}_{>0} \ni \sigma \mapsto\left(i_{\nu} \mapsto f\right)=\left(\left.\sigma \nabla_{\nu} u \mapsto u\right|_{\partial \Omega}\right)
$$

is linear in the resistivity coefficient $\rho=\frac{1}{\sigma}$. Assume that for $\sigma \equiv \sigma_{0}>0$, we apply current densities $i_{\nu}^{(m)}, m=1, \ldots, M$, on $\partial \Omega$, and for each current we consider $L \in \mathbb{N}$ point-evaluations $U^{(m)} \in \mathbb{R}_{\diamond}^{L}$ of the boundary potential $f^{(m)}$. Likewise, denote by $\mathcal{V}=\left(V^{(1)}, \ldots, V^{(M)}\right)$ another set of the potential evaluations under unit-conductivity $\sigma \equiv 1$ for the same currents. Then, exploiting the linearity in $\frac{1}{\sigma_{0}}$, we get the initial guess

$$
\sigma_{0}^{\operatorname{cont}}(\mathcal{I}, \mathcal{U}):=\frac{\sum_{l=1}^{L} \sum_{m=1}^{M}\left(V_{l}^{(m)}\right)^{2}}{\sum_{l=1}^{L} \sum_{m=1}^{M} U_{l}^{(m)} V_{l}^{(m)}}
$$


which minimizes the functional

$$
\sum_{l=1}^{L} \sum_{m=1}^{M}\left(U_{l}^{(m)}-\frac{1}{\sigma_{0}} V_{l}^{(m)}\right)^{2}
$$

see [4. When approximating the continuum model with electrode measurements, $U^{(m)}$ are measured electrode potentials for current densities $i_{\nu}^{(m)}$ which are generated by current vectors $I^{(m)}, m=1, \ldots, M$.

Shunt model approximation. To motivate a CEM variant, we first consider the shunt electrode model which is free of contact impedances and assumes that $u \equiv U_{l}$ on each electrode $E_{l}$ for $l=1, \ldots, L$, and $i_{\nu}=0$ on the gaps. Green's identity for harmonic functions yields

$$
\int_{\Omega} \nabla u \nabla v \mathrm{~d} x=\int_{\partial \Omega} v \nabla_{\nu} u \mathrm{~d} S=\int_{\partial \Omega} u \nabla_{\nu} v \mathrm{~d} S
$$

Here, $u$ and $v$ are solutions to Laplace's equation (2.1) with $\sigma \equiv \sigma_{0}$ and $\sigma \equiv 1$, respectively, for identical Neumann data, that is $i_{\nu}=\sigma_{0} \frac{\partial u}{\partial \nu}=\frac{\partial v}{\partial \nu}$. Solving for $\sigma_{0}$ and averaging over $M$ current patterns, the resulting conductivity estimate reads

$$
\sigma_{0}=\frac{1}{M} \sum_{m=1}^{M} \frac{\int_{\partial \Omega} v^{(m)} i_{\nu}^{(m)} \mathrm{d} S}{\int_{\partial \Omega} u^{(m)} i_{\nu}^{(m)} \mathrm{d} S}=\frac{1}{M} \sum_{m=1}^{M} \frac{\sum_{l=1}^{L} V_{l}^{(m)} I_{l}^{(m)}}{\sum_{l=1}^{L} U_{l}^{(m)} I_{l}^{(m)}}=: \sigma_{0}^{\text {shunt }}(\mathcal{I}, \mathcal{U}),
$$

where $I_{l}^{(m)}=\int_{E_{l}} i_{\nu}^{(m)} \mathrm{d} S$ is an applied electrode current.

CEM approximation. Because of the aforementioned potential drop at the electrode surface, the conductivity estimates of $(3.2)$ and $(3.3)$ become increasingly inaccurate for the CEM as the contact impedance increases. We propose a modified version of equation $(3.3)$, incorporating the electrode contact impedance. In the CEM, we have on each electrode $E_{l}$ that

$$
u+z_{l} i_{\nu}=U_{l} \quad \text { and } \int_{E_{l}} i_{\nu} \mathrm{d} S=I_{l}, \quad l=1, \ldots, L,
$$

while $i_{\nu}=0$ on the gaps [19. Assuming that the interior potential $u$ is approximately constant along each electrode, we get $u=U_{l}-z_{l} i_{\nu} \approx U_{l}-z_{l}\left|E_{l}\right|^{-1} I_{l}$, and substitute this term in (3.3) for $u^{(m)}$ and $v^{(m)}$, respectively. Then, the CEM approximation of $(3.3)$ reads

$$
\sigma_{0} \approx \frac{1}{M} \sum_{m=1}^{M} \frac{\sum_{l=1}^{L}\left(V_{l}^{(m)}-z_{l}\left|E_{l}\right|^{-1} I_{l}^{(m)}\right) I_{l}^{(m)}}{\sum_{l=1}^{L}\left(U_{l}^{(m)}-z_{l}\left|E_{l}\right|^{-1} I_{l}^{(m)}\right) I_{l}^{(m)}}=: \sigma_{0}^{\mathrm{CEM}}(\mathcal{I}, \mathcal{U}) .
$$

Table 1 lists the conductivity estimates $\sigma_{0}^{\text {cont }}, \sigma_{0}^{\text {shunt }}$ and $\sigma_{0}^{\text {CEM }}$ from CEM data for various contact impedances. The estimates of $\sigma_{0}^{\mathrm{CEM}}$ are accurate even for very high contact impedances, where the continuum and shunt approximations fail.

\begin{tabular}{lrrrrr}
\hline$z_{l} \equiv$ & 0.001 & 0.01 & 0.1 & 1 & 10 \\
\hline$\sigma_{0}^{\text {cont }}$ & 0.251 & 0.266 & 0.367 & 0.736 & 0.962 \\
$\sigma_{0}^{\text {shunt }}$ & 0.251 & 0.268 & 0.375 & 0.739 & 0.962 \\
$\sigma_{0}^{\text {CEM }}$ & 0.250 & 0.253 & 0.255 & 0.251 & 0.255 \\
\hline
\end{tabular}

TABLE 1. Conductivitiy estimates for a wide range of contact impedances on the unit disk with conductivity $\sigma \equiv 0.25$ and 16 equally spaced electrodes covering $50 \%$ of the boundary. Data was simulated using the FEM for a full set of normalized adjacent current patterns. 
Contact impedance estimation. The contact impedances are usually unknown. If they can be assumed to be constant, $z_{l} \equiv z>0, l=1, \ldots, L$, then they can be estimated along with the background conductivity with the above method. Denoting by $w>0$ an arbitrary contact impedance for the simulated data $\mathcal{V}$, we get from $(3.4)$ a linear system of equations in $z$ and $\rho_{0}=\frac{1}{\sigma_{0}}$ as

$$
\begin{gathered}
a_{m} \rho_{0}+b_{m} z=c_{m}, \quad m=1, \ldots, M, \quad \text { where } \\
a_{m}=\sum_{l=1}^{L}\left(V_{l}^{m}-w\left|E_{l}\right|^{-1} I_{l}^{m}\right) I_{l}^{m}, \quad b_{m}=\sum_{l=1}^{L}\left|E_{l}\right|^{-1}\left(I_{l}^{m}\right)^{2}, \quad c_{m}=\sum_{l=1}^{L} U_{l}^{m} I_{l}^{m} .
\end{gathered}
$$

Then, the estimates are $\sigma_{0}^{\mathrm{CEM}, z}:=\left(\widehat{\rho}_{0}\right)^{-1}$ and $z_{0}^{\mathrm{CEM}, z}:=\widehat{z}$, where $\widehat{\rho}_{0}$ and $\widehat{z}$ are the least-squares solutions of the linear regression problem (3.5). However, one must take care that the system is not under-determined. This is the case, for example, for a rotation-invariant setting and a rotational set of current patterns, like adjacent currents, in which case $a_{m}, b_{m}$ and $c_{m}$ are constant in $m$. Instead, one can use reduced discrete cosine ${ }^{2}$ or Haar ${ }^{3}$ basis currents, or linearly combine adjacent measurements to remove rotation invariance.

Choosing $w$ small improves the assumption of almost constant interior potentials in the nominator of (3.4). Table 2 lists estimates for various contact impedances: Rows 1-2 show estimates for constant contact impedances, rows 3-4 show estimates for contact impedances varying by $\pm 10 \%$, i.e.

$$
z_{l}=z+0.1 z n_{l}
$$

where $n_{l} \sim u[-1,1]$ are independent uniformly distributed numbers for $l=1, \ldots, L$.

\begin{tabular}{clrrrrr}
\hline variance & $z$ & $1.00 \mathrm{e}-3$ & $1.00 \mathrm{e}-2$ & $1.00 \mathrm{e}-1$ & 1.00 & $1.00 \mathrm{e}+1$ \\
\hline \multirow{2}{*}{$0 \%$} & $z_{0}^{\text {CEM,z }}$ & $7.15 \mathrm{e}-4$ & $1.61 \mathrm{e}-2$ & $1.20 \mathrm{e}-1$ & 1.03 & $1.00 \mathrm{e}+1$ \\
& $\sigma_{0}^{\text {CEM,z }}$ & 0.250 & 0.250 & 0.247 & 0.243 & 0.242 \\
\hline \multirow{2}{*}{ $\pm 10 \%$} & $z_{0}^{\text {CEM,z }}$ & $6.30 \mathrm{e}-4$ & $1.57 \mathrm{e}-2$ & $1.19 \mathrm{e}-1$ & 1.05 & $9.99 \mathrm{e}+0$ \\
& $\sigma_{0}^{\text {CEM,z }}$ & 0.250 & 0.250 & 0.247 & 0.243 & 0.242 \\
\hline
\end{tabular}

TABLE 2. Conductivitiy and contact impedance estimates for a wide range of contact impedances. Setting as in Table 1. Potentials from adjacent currents are linearly transformed to the Haar basis (results for cosine currents are almost identical). The contact impedance for the reference data $\mathcal{V}$ is $w=10^{-3}$.

Note that (3.5) has at most $M \leq L$ degrees of freedom, so estimating $\sigma_{0}$ and all individual contact impedances $z_{l}, l=1, \ldots, L$, simultaneously is not directly possible, but $\sigma_{0}^{\mathrm{CEM}, z}$ and $z_{0}^{\mathrm{CEM}, z}$ can be useful initial guesses for other estimation techniques; see [21. However, (3.5) can be easily extended to estimate each single contact impedance in turn, which can be useful for detecting bad contacts of a measurement setup. We do not carry out this modification here.

3.2. Noise level estimation. As the ICP is ill-posed, measurement noise is a main reason for its instability, and knowing the total measurement error

$$
\delta:=\left\|\mathcal{U}^{v}-\mathcal{U}\right\|_{\text {Fro }}
$$

for a set of noisy measurements $\mathcal{U}^{v}$ is helpful for applying regularization. In practice, $\delta$ is unknown, but we can observe the effect of the noise on the symmetry of the ND data. For noiseless ND data $(\mathcal{I}, \mathcal{U})$, where $\mathcal{I}$ forms a basis of $\mathbb{R}_{\diamond}^{L}$ and $\mathcal{I}^{+}$denotes its pseudo-inverse, the

\footnotetext{
${ }^{2}$ Discrete cosine transform basis, omitting the constant vector. Provided by the rows of dctmtx in MATLAB.

${ }^{3}$ Discrete Haar-wavelet basis, omitting the constant vector. $L$ must be a power of 2 .
} 
matrix $\mathcal{U I}^{+}=F(\sigma)$ is symmetric. However, for potentials perturbed by (additive) noise, that is,

$$
\mathcal{U}^{v}=\mathcal{U}+N^{v}
$$

for some noise matrix $N^{v} \in \mathbb{R}^{L \times M}$, we observe a symmetry error

$$
e_{v}:=\left\|\mathcal{U}^{v} \mathcal{I}^{+}-\left(\mathcal{U}^{v} \mathcal{I}^{+}\right)^{\top}\right\|_{\text {Fro }}^{2}
$$

Assuming that all entries of $N^{v}$ are independent and identically distributed (iid) with zero mean and variance $v>0$, we show in Appendix A that the expected value of the symmetry error is

$$
\mathbb{E} e_{v}=2(L-1)\left\|\mathcal{I}^{+}\right\|_{\text {Fro }}^{2} v .
$$

If the noise is Gaussian, i.e. $N_{l, m}^{v} \stackrel{\text { iid }}{\sim} \mathcal{N}(0, v)$ for $l=1, \ldots, L, m=1, \ldots, M$, the expected value of the measurement error $\delta$ is

$$
\mathbb{E} \delta=\mathbb{E}\left\|N^{v}\right\|_{\text {Fro }}=\sqrt{2} \frac{\Gamma\left(\frac{M L+1}{2}\right)}{\Gamma\left(\frac{M L}{2}\right)} \sqrt{v} \approx \sqrt{M L v}
$$

with Euler's gamma function $\Gamma$; see standard literature. Replacing the expected value of the symmetry error in (3.7) by the observed one $\left(e_{v}\right)$ and substituting (3.8) into (3.7), we get an estimator of the total measurement error by

$$
\delta^{\mathrm{CEM}}\left(\mathcal{I}, \mathcal{U}^{v}\right):=\sqrt{\frac{M L}{2(L-1)}} e_{v}\left\|\mathcal{I}^{+}\right\|_{\text {Fro }}^{-1} \approx \mathbb{E} \delta .
$$

Testing the estimator. To evaluate the quality of the estimator, we simulate noisy data $\mathcal{U}^{v}$ and compare the true data error $\delta$ with the estimate $\delta^{\mathrm{CEM}}$. In principle, we could specify a variance $v>0$ explicitly and generate a realization of additive noise $N^{v}$. However, for a single measurement $U^{v} \in \mathbb{R}_{\diamond}^{L}$, the typical error $\left\|U^{v}-U\right\|_{2}$ of a measurement system is often proportional to the magnitude $\left\|U^{v}\right\|_{2}$ of the measurement. To generate "meaningful" noise in our numerical experiments for an average relative measurement precision $\eta>0$, we thus determine a variance $v$ such that

$$
\eta \approx \mathbb{E} \frac{\left\|\left(U^{v}\right)^{(m)}-U^{(m)}\right\|_{2}}{\left\|U^{(m)}\right\|_{2}}=\frac{1}{\left\|U^{(m)}\right\|_{2}} \mathbb{E}\left\|\left(N^{v}\right)^{(m)}\right\|_{2}=\frac{\sqrt{2 v}}{\left\|U^{(m)}\right\|_{2}} \frac{\Gamma\left(\frac{L+1}{2}\right)}{\Gamma\left(\frac{L}{2}\right)}, m=1, \ldots, M .
$$

Assuming that all potentials have roughly the same norm, $\left\|U^{(m)}\right\|_{2} \approx \frac{1}{M} \sum_{i=1}^{M}\left\|U^{(i)}\right\|_{2}$, we thus set

$$
v=v(\eta):=\frac{1}{2}\left(\frac{\eta \Gamma\left(\frac{L}{2}\right) \sum_{m=1}^{M}\left\|U^{(m)}\right\|_{2}}{M \Gamma\left(\frac{L+1}{2}\right)}\right)^{2}
$$

for a given $\eta>0$. With the noise model

$$
\mathcal{U}^{v}=\mathcal{U}+N^{v(\eta)}, \quad N_{l, m}^{v(\eta)} \stackrel{\operatorname{iid}}{\sim} \mathcal{N}(0, v(\eta)) \quad \text { for } \quad l=1, \ldots, L, \quad m=1, \ldots, M,
$$

we simulate noisy data and validate the accuracy of our error estimator $\delta^{\text {CEM }}$. Table 3 lists the true error $\delta$ and the estimated error $\delta^{\mathrm{CEM}}$ from single realizations of $N^{v(\eta)}$ for various $\eta$ and current patterns. All estimates are within relative distance $\left|1-\delta / \delta^{\mathrm{CEM}}\right|<5 \%$ of the true error. This will be relevant for defining a stopping rule of our Algorithm in section 3.6. 


\begin{tabular}{cccccccc}
\hline$\eta$ & $\delta_{\text {adjacent }}$ & $\delta_{\text {adjacent }}^{\text {CEM }}$ & $\delta_{\text {cosine }}$ & $\delta_{\text {cosine }}^{\text {CEM }}$ & $\delta_{\text {Haar }}$ & $\delta_{\text {Haar }}^{\text {CEM }}$ & $\left|1-\delta / \delta^{\text {CEM }}\right|$ \\
\hline $1.0 \mathrm{e}-4$ & $1.34 \mathrm{e}-3$ & $1.32 \mathrm{e}-3$ & $1.85 \mathrm{e}-3$ & $1.77 \mathrm{e}-3$ & $1.85 \mathrm{e}-3$ & $1.93 \mathrm{e}-3$ & $<5 \%$ \\
$1.0 \mathrm{e}-3$ & $1.36 \mathrm{e}-2$ & $1.42 \mathrm{e}-2$ & $1.85 \mathrm{e}-2$ & $1.79 \mathrm{e}-2$ & $1.93 \mathrm{e}-2$ & $1.87 \mathrm{e}-2$ & $<5 \%$ \\
$1.0 \mathrm{e}-2$ & $1.30 \mathrm{e}-1$ & $1.29 \mathrm{e}-1$ & $1.75 \mathrm{e}-1$ & $1.81 \mathrm{e}-1$ & $1.83 \mathrm{e}-1$ & $1.82 \mathrm{e}-1$ & $<4 \%$ \\
$1.0 \mathrm{e}-1$ & $1.35 \mathrm{e}-0$ & $1.29 \mathrm{e}-0$ & $1.82 \mathrm{e}-3$ & $1.79 \mathrm{e}-0$ & $1.91 \mathrm{e}-0$ & $1.99 \mathrm{e}-0$ & $<5 \%$ \\
\hline
\end{tabular}

TABLE 3. Noise estimates $\delta^{\mathrm{CEM}}$ for simulated data with additive pseudo-random normal noise of variance $v(\eta)$ using adjacent, cosine and Haar current patterns. Setting as in Table 1 .

3.3. Constant conductivity considerations. The ICP is a constrained $(\sigma>0)$ and highly nonlinear problem. This can lead to several problems for Newton-type algorithms as they solve unconstrained problems by linearization. Firstly, a Newton update can result in negative conductivities or - if the step size is reduced accordingly - in very slow convergence. This is particularly bad since the nonlinearity of the forward map and thus the linearization error increases as the conductivity approaches zero (Algorithm 1, line 4). Our numerical examples will illustrate this effect (Figure 3a).

A classical and popular method to obtain an unconstrained problem is to recover the logarithm of the conductivity $\log \sigma$, or $-\log \sigma=\log \frac{1}{\sigma}$; see [2]. This approach is also motivated by the filtered back-projection approach for EIT [20].

To improve the convergence speed of a Newton-type method and to increase its convergence radius, we aim to reduce the nonlinearity of the ICP. Although recovering non-constant conductivities in the CEM is always a nonlinear problem, we are encouraged by the fact that recovering a constant conductivity in the continuum model can be transformed to the linear problem of recovering $\rho=\frac{1}{\sigma}$; see (3.1). When recovering inclusions in a constant background, ND data is most sensitive to the background conductivity, thus improving the linearity with respect to the background conductivity is reasonable. The improved linearity is particularly useful when recovering inclusions in an unknown constant background by linear (one-step) methods. This has been investigated in detail for lung EIT [9], where reconstructions of $\sigma, \log \sigma$ and $\rho$ with the (one-step) NOSER algorithm [4] are compared. However, recovering the resistivity is again a constrained problem $(\rho>0)$. Conversely to the conductivity case, linearization error increases for very large conductivities in the non-constant (nonlinear) case.

Coefficient transformation. Instead of linearizing $F$ directly, we want to apply the Newton step to a transformation of the conductivity coefficient. To that end, we consider injective $C^{1}$-transformations

$$
t_{*}:(0, \infty) \rightarrow \mathbb{R}, \quad \sigma \mapsto t_{*}(\sigma)=: t,
$$

and their corresponding transformed forward operators defined as

$$
F_{*}\left(t_{*}(\sigma)\right)=F(\sigma), \quad \text { that is, } \quad F_{*}(t)=F\left(t_{*}^{-1}(t)\right)=: F\left(\sigma_{*}(t)\right) .
$$

We first investigate the constrainedness and linearity of transformed forward operators $\mathcal{F}_{*}$, i.e. in the continuum setting defined analogeously to (3.12), for constant conductivities. Although the following relations do not hold for non-constant conductivities or for the CEM, they are still helpful in reducing the nonlinearity of the CEM since it behaves similar to the continuum model for small contact impedances, and since the measured potentials are most sensitive to the background conductivity. Considering $\sigma>0$ as a constant function on $\Omega$, we have that

$$
\mathcal{F}(\sigma)=\frac{1}{\sigma} \mathcal{F}(1), \quad \text { thus } \quad \mathcal{F}^{\prime}(\sigma)=\frac{-1}{\sigma^{2}} \mathcal{F}(1) \quad \text { (in a slight abuse of notation) }
$$

for the derivative in unit direction. Under transformation, we get

$$
\mathcal{F}_{*}^{\prime}(t)=\left[\mathcal{F}\left(\sigma_{*}(t)\right)\right]^{\prime}=\frac{-\sigma_{*}^{\prime}(t)}{\sigma_{*}^{2}(t)} \mathcal{F}(1)=: s_{*}(t) \mathcal{F}(1) .
$$


In that sense, $\left|s_{*}^{\prime}\left(t_{*}(\sigma)\right)\right|$ is a measure of the nonlinearity of $\mathcal{F}_{*}$ at conductivity $\sigma$ : If $s_{*}^{\prime}=0$, then $s_{*}$ and $\mathcal{F}_{*}^{\prime}$ are constant, thus $\mathcal{F}_{*}$ is linear. Table 4 lists the relevant properties of the aforementioned transformations $t_{\mathrm{Id}}(\sigma)=\sigma, t_{\log }(\sigma)=-\log (\sigma)$ and $t_{\rho}(\sigma)=\frac{1}{\sigma}$. We observe, as expected, that $\mathcal{F}_{\mathrm{Id}}=\mathcal{F}$ is highly nonlinear for small conductivities and asymptotically linear for very high conductivities. $\mathcal{F}_{\log }$ is also nonlinear, although of lower order, and leads to an unconstrained inverse problem. Finally, recovering constant resistivities by $\mathcal{F}_{\rho}$ is a linear, although constrained problem.

\begin{tabular}{|c|c|c|c|c|}
\hline * & $\left|s_{*}^{\prime}\left(t_{*}(\sigma)\right)\right|$ & $\overline{\sigma_{*}(t)}$ & $\overline{\sigma_{*}^{\prime}\left(t_{*}(\sigma)\right)}$ & $\overline{t_{*}((0, \infty))}$ \\
\hline $\begin{array}{l}\text { Id } \\
\log \\
\rho\end{array}$ & $\begin{array}{l}2 \sigma^{-3} \\
\sigma^{-1} \\
0\end{array}$ & $\begin{array}{l}t \\
e^{-t} \\
t^{-1}\end{array}$ & $\begin{array}{l}1 \\
-\sigma \\
-\sigma^{2}\end{array}$ & $\begin{array}{l}(0, \infty) \\
(\infty,-\infty) \\
(\infty, 0)\end{array}$ \\
\hline$\alpha$ & $\frac{2 \alpha \sigma^{3}}{\left(1+\alpha\left(\sigma^{2}-1\right)\right)^{3}}$ & $\frac{\sqrt{4 \alpha(1-\alpha)+t^{2}}-t}{2 \alpha}$ & $\frac{-\sigma^{2}}{(1-\alpha)+\alpha \sigma^{2}}$ & $(\infty,-\infty)$ \\
\hline
\end{tabular}

TABLE 4. Properties (nonlinearity, inverse map, Jacobian amplification and range) of various conductivity transformations.

Our goal is to combine both advantages and to obtain an unconstrained problem with limited nonlinearity. To that end, we introduce the transformation

$$
t_{\alpha}(\sigma):=\frac{1-\alpha}{\sigma}-\alpha \sigma
$$

for some parameter $\alpha \in(0,1)$. Being the sum of two strictly decreasing $C^{1}$ functions on $(0, \infty)$, $t_{\alpha}$ is clearly injective. Moreover, the nonlinearity of its corresponding forward operator $\mathcal{F}_{\alpha}$ is bounded, see Table 4. The parameter $\alpha$ is a trade-off between reconstructing the resistivity $(\alpha \rightarrow 0)$ and the conductivity $(\alpha \rightarrow 1)$. It controls the maximum nonlinearity and its occurrence:

$$
\max _{\sigma \in(0, \infty)}\left|s_{\alpha}^{\prime}\left(t_{\alpha}(\sigma)\right)\right|=s_{\alpha}^{\prime}(0)=\frac{\alpha}{4(\alpha(1-\alpha))^{\frac{3}{2}}}, \underset{\sigma \in(0, \infty)}{\arg \max }\left|s_{\alpha}^{\prime}\left(t_{\alpha}(\sigma)\right)\right|=\sqrt{\alpha^{-1}-1} .
$$

The maximum nonlinearity is minimized at $\widehat{\alpha}=0.25$, so in a sense, this is an optimal choice. Figure $1 \mathrm{a}$ shows the transformations $-t_{\mathrm{Id}}, t_{\log }, t_{\rho}$, and $t_{\widehat{\alpha}}$. Figure $1 \mathrm{~b}$ shows various nonlinearity distributions. In the following, we will consider these transformations for the CEM.

Transformation of the Newton step. To solve the linearized equation (Algorithm 1, line 4) in the transformed setting, we need to compute the transformed sensitivity matrix $\mathcal{S}_{*}$ defined by the Fréchet derivative of $F_{*}$. Using the chain rule, we get the Jacobian - evaluated in the $p$ th unit direction of the discretized conductivity space- by

$$
\left.\frac{\partial F_{*}(t)}{(\partial t)_{p}}\right|_{t_{*}(\sigma)}=\left.\frac{\partial F\left(\sigma_{*}(t)\right)}{(\partial t)_{p}}\right|_{t_{*}(\sigma)}=\left.\left.\frac{\partial F(\sigma)}{(\partial \sigma)_{p}}\right|_{\sigma} \frac{\partial \sigma_{*}(t)}{(\partial t)_{p}}\right|_{t_{*}(\sigma)}=\left[F^{\prime}(\sigma)\right]_{p}\left(\sigma_{*}^{\prime}\left(t_{*}(\sigma)\right)\right)_{p} .
$$

Table 4 lists $\sigma_{*}^{\prime}\left(t_{*}(\sigma)\right)$ for the aforementioned transformations. In the transformed setting, lines 35 of Algorithm 1 are substituted by lines $3_{*}-5_{*}$ of Algorithm 2 , respectively.

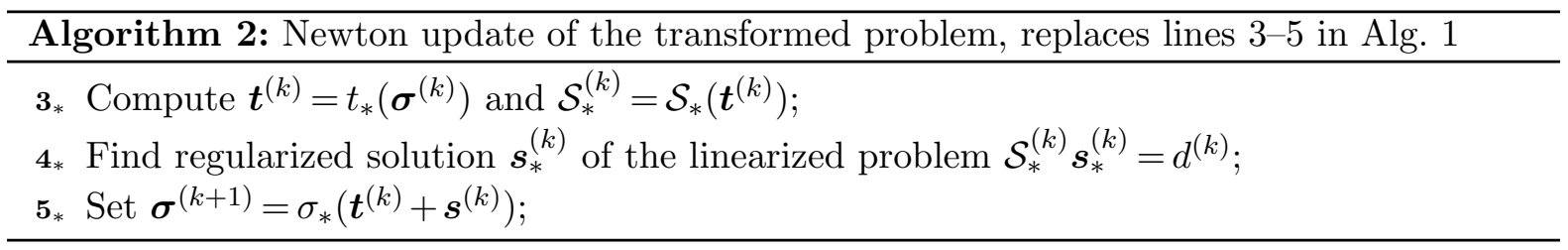




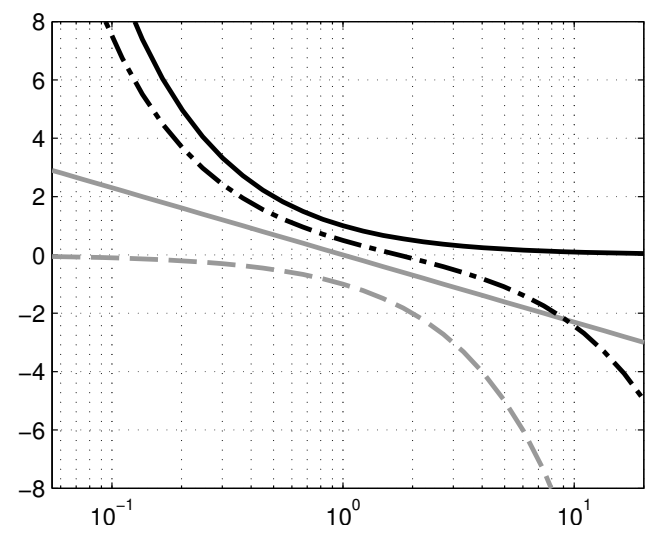

(a) Conductivity transformations $-t_{\text {Id }}(--)$, $t_{\log }(-), t_{\rho}(-)$, and $t_{\widehat{\alpha}}(--)$ on a logarithmic conductivity scale. $t_{\widehat{\alpha}}$ behaves like $t_{\rho}$ for small conductivities and like $-t_{\mathrm{Id}}$ for large conductivities.

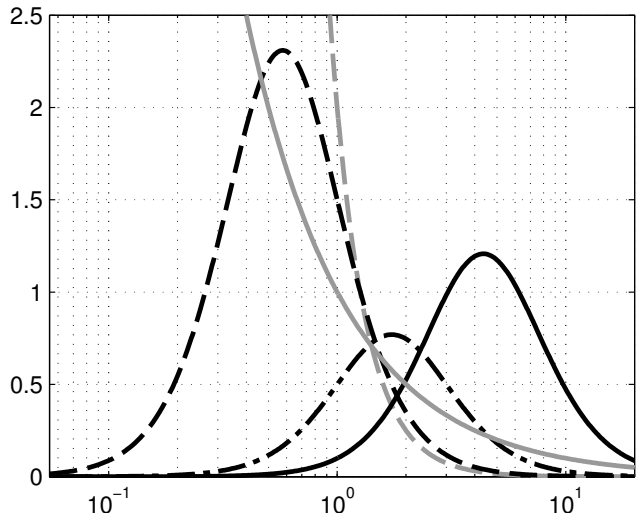

(b) Nonlinearity distributions $\left|s_{\log }^{\prime}\left(t_{\log }(\cdot)\right)\right|(-)$ and $\left|s_{\alpha}^{\prime}\left(t_{\alpha}(\cdot)\right)\right|$ for $\alpha=1(--), \alpha=0.75$ (--), $\alpha=\widehat{\alpha}(--)$, and $\alpha=0.05$ (-).

Note that (--) has the smallest maximum, (- - ) approaches (--) as $\alpha \rightarrow 1$ and (-) approaches 0 pointwise as $\alpha \rightarrow 0$ (the peak moves to infinity).

FiguRE 1. Transformation properties on a logarithmic conductivity scale.

\subsection{Non-constant conductivity considerations. The linearized problem}

$$
\text { Find } \quad \eta_{*} \in L^{\infty}(\Omega) \quad \text { satisfying } \quad F_{*}^{\prime}\left(t_{*}(\sigma)\right)\left[\eta_{*}\right] \mathcal{I}=\mathcal{U}-F_{*}\left(t_{*}(\sigma)\right) \mathcal{I}
$$

is highly under-determined and severely ill-posed. We solve its discretization

$$
\mathcal{S}_{*} \boldsymbol{s}_{*}=d, \quad \boldsymbol{s}_{*} \in \mathbb{R}^{P}, \quad \boldsymbol{s}_{*} \widehat{=} s_{*} \in \mathcal{D}_{\mathcal{P}},
$$

in each Newton step. A regularization strategy to handle the ill-posedness is discussed in section 3.5. To resolve the under-determinedness of (3.15), we introduce a prior assumption on the conductivity. For the untransformed case $\mathcal{S}_{*}=\mathcal{S}$, we consider the case of two columns $p, q$ of $\mathcal{S}$ being collinear, that is, $\mathcal{S}_{q}=\beta \mathcal{S}_{p}$ for some $\beta \in \mathbb{R} \backslash\{0\}$. Then, the linear combination of unit vectors $e_{q}-\beta e_{p}$ is in the Null space of $\mathcal{S}$. This means we have (at least) one degree of freedom in choosing $\boldsymbol{s}_{q}$ versus $\boldsymbol{s}_{p}$ in the solution of (3.15). Physically, the conductivity is a material property represented by a piecewise constant function. Starting from a constant initial guess, it is thus reasonable to update indistinguishable coefficients by the same amount. We postulate this in the following prior:

Reconstruction prior. Assume that $\mathcal{S}_{q}=\beta \mathcal{S}_{p}$ for some $p, q \in\{1, \ldots, P\}$ and $\beta \in \mathbb{R} \backslash\{0\}$, i.e. (3.15) is under-determined in the coefficients $\boldsymbol{s}_{\{p, q\}}$ for all $d \in \mathbb{R}^{L M}$. Then, $\boldsymbol{s}_{\{p, q\}}$ should be chosen proportional to their local conductivities $\sigma_{\{p, q\}}$, that is, $\frac{s_{p}}{\sigma_{p}}=\frac{\operatorname{sign}(\beta) s_{q}}{\sigma_{q}}$.

The proportionality of the update to the local conductivity value is motivated by the inverse proportionality of the forward operator for constant conductivities (3.13).

Reconstruction prior and the pseudo-inverse. We will first consider the untransformed setting $F_{*}=F$. A popular solution strategy for $(3.15)$ is to approximate the Moore-Penrose pseudoinverse

$$
\boldsymbol{s}^{+}=\boldsymbol{s}^{+}(d)=\mathcal{S}^{+} d=\underset{\boldsymbol{s} \in \mathcal{N}(\mathcal{S})^{\perp}}{\arg \min }\|\mathcal{S} \boldsymbol{s}-d\|_{2},
$$

where the condition $s \in \mathcal{N}(\mathcal{S})^{\perp}$ resolves the under-determinedness. However, $\mathcal{N}(\mathcal{S})^{\perp}$ depends on the scaling of each column of $\mathcal{S}$, which in turn depends on the local sensitivity and the (arbitrary) choice of the conductivity update discretization space $\mathcal{D}_{\mathcal{P}}$. Fortunately, 3.16$)$ can be modified to reflect the reconstruction prior by introducing a weighted inner product on the 
conductivity update coefficient space $\mathbb{R}^{P}$. To that end we denote, for $0<W \in \mathbb{R}^{P \times P}$, a weighted inner product and its corresponding norm by

$$
\left\langle\cdot \cdot_{1}, \cdot_{2}\right\rangle_{W}:=\left\langle\cdot \cdot_{1}, W \cdot \cdot_{2}\right\rangle_{\mathbb{R}^{P}} \quad \text { and } \quad\|\cdot\|_{W}=\left\|W^{\frac{1}{2}} \cdot\right\|_{2},
$$

respectively, and define a weighted pseudo-inverse by

$$
\boldsymbol{s}^{+W}=\boldsymbol{s}^{{ }_{W}}(d)=\mathcal{S}^{+{ }_{W}} d=\underset{s \in \mathcal{N}(\mathcal{S})^{\perp_{W}}}{\arg \min }\|\mathcal{S}-d\|_{2},
$$

where $\perp_{W}$ denotes orthogonality with respect to the weighted inner product. The effect of a diagonal weighting matrix on the pseudo-inverse solution is summarized in the following theorem, which is proved in Appendix B.1.

Theorem 1. Consider the situation of (3.16) and (3.17) and assume that $\mathcal{S}_{q}=\beta \mathcal{S}_{p}$ for some $\beta \in \mathbb{R} \backslash\{0\}$. Further, let $W=\operatorname{diag}\left(w_{1}, \ldots, w_{P}\right)>0$. Then, we have the following proportionality relations for all $d \in \mathbb{R}^{L M}$ :

(a) $\boldsymbol{s}_{q}^{+}(d)=\beta \boldsymbol{s}_{p}^{+}(d)$,

(b) $\frac{w_{p}}{\left\|\mathcal{S}_{p}\right\|_{2}} \boldsymbol{s}_{p}^{+W}(d)=\frac{\operatorname{sign}(\beta) w_{q}}{\left\|\mathcal{S}_{q}\right\|_{2}} \boldsymbol{s}_{q}^{+W}(d)$,

An immediate consequence of Theorem $1 \mathrm{~b}$ is

Corollary 2. For

$$
W=W_{\mathcal{S}, \sigma}:=\operatorname{diag}\left(\left\|\mathcal{S}_{1}\right\|_{2} \boldsymbol{\sigma}_{1}^{-1}, \ldots,\left\|\mathcal{S}_{P}\right\|_{2} \boldsymbol{\sigma}_{P}^{-1}\right),
$$

the weighted pseudo-inverse satisfies the reconstruction prior.

Roughly speaking, the weighting can be used to "stretch" orthogonality to compensate for the scaling differences of the columns of $\mathcal{S}$. As the pseudo-inverse is unique, we resolve underdeterminedness and satisfy the reconstruction prior simultaneously by choosing $\boldsymbol{s}^{+W_{\mathcal{S}, \boldsymbol{\sigma}}}$ as the Newton update. We also get the following estimate for almost collinear columns of $\mathcal{S}$, which is proved in Appendix B.2.

Corollary 3. Assume that $\mathcal{S}_{p}$ and $\mathcal{S}_{q}$ are almost collinear, that is $\mathcal{S}_{q}=\beta \mathcal{S}_{p}+\kappa \boldsymbol{k}, \mathcal{S}_{p} \perp \boldsymbol{k} \in \mathbb{R}^{P}$, $\|\boldsymbol{k}\|_{2}=1, \beta \in \mathbb{R} \backslash\{0\}, \kappa>0$. Then,

$$
\left|\frac{w_{p}}{\left\|\mathcal{S}_{p}\right\|_{2}} \boldsymbol{s}_{p}^{+W}(d)-\frac{\operatorname{sign}(\beta) w_{q}}{\left\|\mathcal{S}_{q}\right\|_{2}} \boldsymbol{s}_{q}^{+W}(d)\right| \leq \sigma_{\min }^{-1}\left(\kappa \sigma_{\min }^{-1}\left\|\mathcal{S}_{q}\right\|^{-1}+c_{\kappa}\right)\|d\|_{2} .
$$

where $\sigma_{\min }$ is the smallest non-zero singular value of $\mathcal{S}$ and $c_{\kappa}=\mathcal{O}\left(\kappa^{2}\right)$ as $\kappa \rightarrow 0$. In particular, the right-hand side vanishes as $\kappa \rightarrow 0$ if and only if $\boldsymbol{k} \in \operatorname{span}\left\{\mathcal{S}_{l}: l \in\{1, \ldots, P\} \backslash q\right\}$.

Note that $\mathcal{S}_{p} \perp \boldsymbol{k}$ is not a restriction since $\boldsymbol{k}$ can be split into orthogonal parts otherwise, and Corollary 3 holds for modified constants $\beta$ and $\kappa$.

For $W=W_{\mathcal{S}, \sigma}$, Corollary 3 states that if two columns of $\mathcal{S}$ are almost collinear relative to the rest of the columns, then their coefficients in the weighted pseudo-inverse are almost proportional to the local conductivity.

In section 4 , we will also investigate the individual impacts of normalizing by the sensitivity and the background conductivity only, using the weights

$$
W_{\mathcal{S}}:=\operatorname{diag}\left(\left\|\mathcal{S}_{1}\right\|_{2}, \ldots,\left\|\mathcal{S}_{P}\right\|_{2}\right) \quad \text { and } \quad W_{\boldsymbol{\sigma}}:=\operatorname{diag}\left(\boldsymbol{\sigma}_{1}^{-1}, \ldots, \boldsymbol{\sigma}_{P}^{-1}\right)
$$

respectively. The positive effect of $W_{\mathcal{S}}$ on conductivity updates has been observed in [5, 18, 14, although without a thorough analysis of the reasons ${ }^{4}$. To our knowledge, using $W_{\mathcal{S}, \sigma}$ is novel.

\footnotetext{
${ }^{4}$ In [18, the improved reconstructions when using the weights $W_{\mathcal{S}}$ are explained by an observed decrease $3 \cdot 10^{17} \rightarrow 1 \cdot 10^{17}$ of the condition of eq. 3.17 . Although improving the condition helps to stabilize the solution, we feel that the main impact of these weights is how they shift orthogonality to the Null space, and thus which types of solutions are promoted in case of under-determinedness.
} 
Sensitivity and discretization. To see the connection between the reconstruction prior and the choice of discretization $\mathcal{P}$, we recall the definition of the sensitivity matrix

$$
\mathcal{S}_{p}=\operatorname{col}\left(\left(F^{\prime}(\sigma)\left[\chi_{\Omega_{p}}\right]\right) \mathcal{I}\right)=\operatorname{col}\left(-\int_{\Omega_{p}} w(x) \mathcal{I} \mathrm{d} x\right), \quad w(x)=\left(\nabla u^{(l)}(x) \nabla u^{(m)}(x)\right)_{l, m=1, \ldots, L} .
$$

For piecewise constant conductivities, 2.1 is the Laplacian on each cell $\Omega_{p}, p=1, \ldots, P$, and thus the sensitivity function $\bar{w}_{\mathcal{I}}(x):=\|w(x) \mathcal{I}\|_{\text {Fro }}$ is piecewise continuous, hence for $\Omega_{p}$ small, we get the approximation $\left\|\mathcal{S}_{p}\right\|_{2} \approx \bar{w}_{\mathcal{I}}\left(x_{p}\right)\left|\Omega_{p}\right|$ for any $x_{p} \in \Omega_{p}$. We can thus interpret the weighting matrices $W_{\mathcal{S}}$ and $W_{\mathcal{S}, \boldsymbol{\sigma}}$ on the conductivity update coefficient space $\mathbb{R}^{P}$ as normalizations with respect to the local mesh size $\left|\Omega_{p}\right|$ and sensitivity $\bar{w}_{\mathcal{I}}\left(x_{p}\right)$.

Thus, the reconstruction prior-incorporated by $W_{\mathcal{S}, \sigma}$-results in unique Newton updates which are independent of the discretization geometry, assuming it is sufficiently fine.

The reconstruction prior in the transformed setting. When reconstructing a transformation $t_{*}$ of the conductivity coefficient, the scaling imposed by the reconstruction prior must be translated into the transformed setting. By the chain rule, the weight $\boldsymbol{\sigma}_{p}^{-1}$ of $\boldsymbol{s}_{p}$ in the conductivity setting is amplified by $\sigma_{*}^{\prime}\left(t_{*}\left(\boldsymbol{\sigma}_{p}\right)\right)$ in the transformed setting. Thus, the transformed weighting matrix reads

$$
W_{\mathcal{S}_{*}, \boldsymbol{\sigma}}=\operatorname{diag}\left\{\sigma_{*}^{\prime}\left(t_{*}\left(\boldsymbol{\sigma}_{\mathbf{1}}\right)\right)\left\|\left(\mathcal{S}_{*}\right)_{1}\right\|_{2} \boldsymbol{\sigma}_{\mathbf{1}}^{-1}, \ldots, \sigma_{*}^{\prime}\left(t_{*}\left(\boldsymbol{\sigma}_{\boldsymbol{P}}\right)\right)\left\|\left(\mathcal{S}_{*}\right)_{P}\right\|_{2} \boldsymbol{\sigma}_{\boldsymbol{P}^{-1}}\right\}
$$

Note that $\left\|\left(\mathcal{S}_{*}\right)_{p}\right\|_{2}=\sigma_{*}^{\prime}\left(t_{*}\left(\boldsymbol{\sigma}_{\boldsymbol{p}}\right)\right)\left\|\mathcal{S}_{p}\right\|_{2}$. An interesting observation is that $\left|\sigma_{\log }^{\prime}\left(t_{\log }(\boldsymbol{\sigma})\right) \boldsymbol{\sigma}^{-1}\right| \equiv 1$. This means that the log-transform inherits the conductivity-scaling implicitly: $W_{\mathcal{S}_{\log }, \sigma}=W_{\mathcal{S}_{\text {log }}}$.

3.5. Ill-posedness and regularization. To account for the ill-posedness of (3.14), we need a regularization strategy when solving the conductivity update equation (3.17) which inherits its ill-posedness. Common approaches are the Landweber regularization, the Tikhonov-Phillips regularization and the truncated singular value decomposition.

Following [16, 12, 22, we will use a (nonlinear) regularized conjugate gradient (cg) method to approximate (3.17) in the transformed setting. The regularization strategy is to stop the pursuit of cg directions "early", when the linear residual is reached to a certain relative tolerance $\theta \in(0,1]$; see Algorithm 3 . Therein, $\mathcal{S}_{*}^{\prime}=\mathcal{S}_{*}^{\top} W_{\mathcal{S}_{*}, \boldsymbol{\sigma}}^{-1}$ is the adjoint matrix of $\mathcal{S}_{*}$ with respect to $\langle\cdot, \cdot\rangle_{W_{\mathcal{S}_{*}, \sigma}}$, i.e. satisfying $x^{\top} \mathcal{S}_{*}^{\prime} W_{\mathcal{S}_{*}, \sigma} y=\left\langle x, \mathcal{S}_{*}^{\prime} y\right\rangle_{W_{\mathcal{S}_{*}, \sigma}}=\left\langle\mathcal{S}_{*} x, y\right\rangle_{\mathbb{R}^{L M}}=x^{\top} \mathcal{S}_{*}^{\top} y$ for all $x \in \mathbb{R}^{P}, y \in$ $\mathbb{R}^{L M}$. The regularization effect of this method was studied in detail in [16, 17].

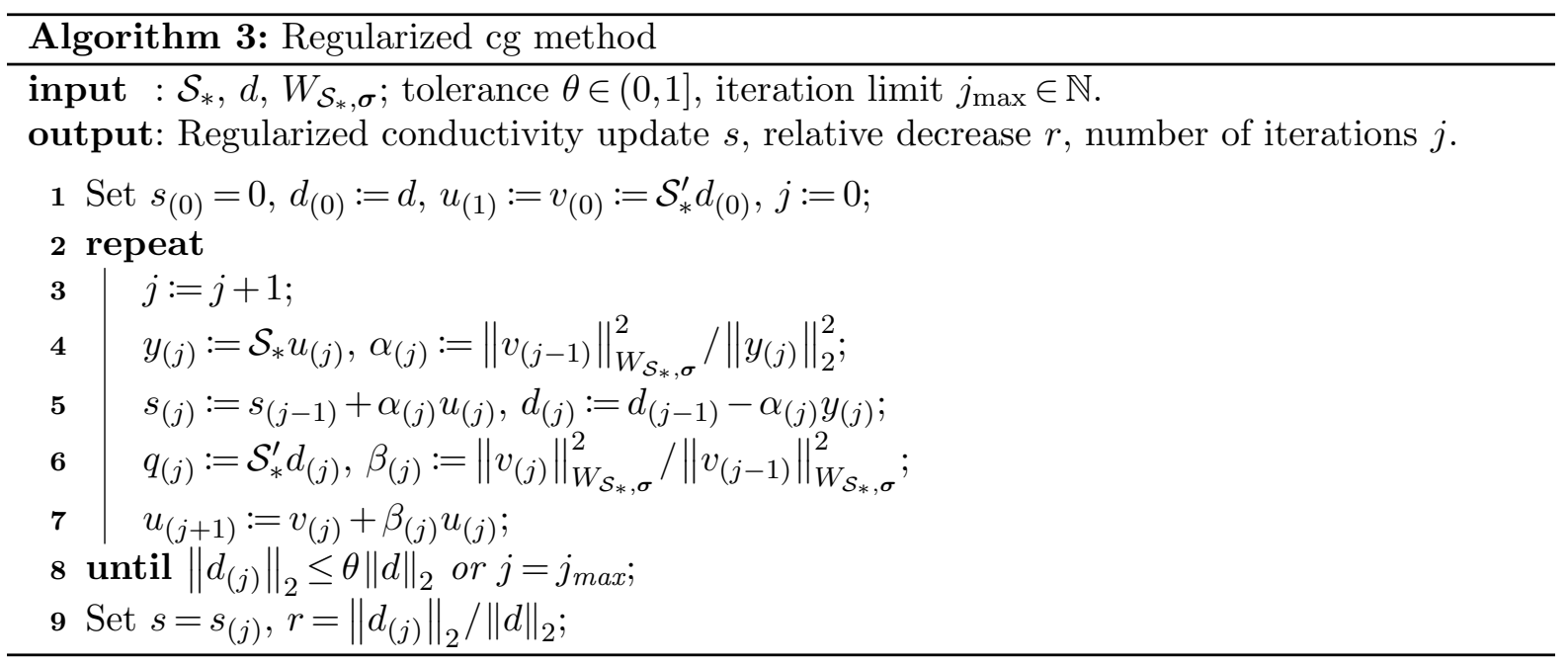

The parameters $\theta=\theta^{(k)}$ and $j_{\max }=j_{\max }^{(k)}$ of Algorithm 3 can be chosen dynamically each time (3.15) is solved, i.e. in the $k$ th iteration of Algorithm (2). For example, the special case 
$j_{\max }^{(k)} \equiv 1$ yields the steepest descent method. We will use the parameter strategy of [16, section $6]$ with some modifications. The first Newton-step suffers most from the nonlinearity of the problem, thus we let $j_{\max }^{(0)}=1, \theta^{(0)}=1$ to maximize regularization. For the next iteration, we set $\theta^{(1)}:=r^{(0)}$ (relative decrease of the previous Newton step) and $j_{\max }^{(1)}:=2$. For $k \geq 2$, we use the parameter strategy of [16] for the tolerances, which yields

$$
\theta^{(k)}:= \begin{cases}1, & k=0, \\ r^{(0)}, & k=1, \\ \theta_{\max }\left(1-\frac{j^{(k-2)}}{j^{(k-1)}}\left(1-\theta^{(k-1)}\right)\right), & k \geq 2 \text { and } j^{(k-1)}>j^{(k-2)}, \\ \theta_{\max \gamma} \theta^{(k-1)}, & \text { otherwise. }\end{cases}
$$

The parameters $\gamma$ and $\theta_{\max }$ should be chosen close to 1 , but the exact choice is uncritical. We let $\gamma:=0.99$ and $\theta_{\max }:=\sqrt{\gamma}$. Further, we set $j_{\max }^{(k)}:=j^{(k-1)}+j^{(k-2)}$ for $k \geq 2$, i.e. we limit the number of cg iterations relative to those in the previous Newton steps. This prevents "iteration peaks" when the Newton method is close to convergence and replaces the safeguarding-technique of [16, section 6]. In all, we have

$$
j_{\max }^{(k)}= \begin{cases}1, & k=0 \\ 2, & k=1, \\ j^{(k-1)}+j^{(k-2)}, & k \geq 2 .\end{cases}
$$

3.6. A model-aware Newton-type inversion scheme. We have now gathered all ingredients for a modification of Algorithm 1 which takes into account model properties of the CEM and a reconstruction prior based on a material property of the conductivity. The whole inversion process is shown in Algorithm 4 (MANTIS). Therein, $\mathcal{U}^{(k)}$ denotes the approximation of $F\left(\sigma^{(k)}\right) \mathcal{I}$ computed by the FEM on a suitabl 5 triangulation $\mathcal{P}$ of $\Omega$, and $\mathcal{S}_{*}^{(k)}$ denotes the corresponding transformed Jacobian approximation (line 1). To avoid instability of the FEM forward solver, we require bounds for the contact impedances $z_{l} \geq z_{\min }, l=1, \ldots, L$, and the conductivity $\sigma_{\min } \leq \sigma \leq \sigma_{\min }^{-1}\left(z_{\min }=\sigma_{\min }:=10^{-4}\right.$ in all of our computations). The MANTIS algorithm terminates by Morozov's discrepancy principle (line 6). The parameter $\tau>1$ should be chosen to reflect the uncertainty of the measurement error estimate $\delta^{\mathrm{CEM}}$. We set $\tau:=1.1$ for all reconstructions as $\delta^{\mathrm{CEM}}$ was within $5 \%$ distance of $\delta$ in all of our tests; see section 3.2 . Thus, we consider $\tau$ as a constant rather than a design parameter, which needs to be increased only if convergence issues occur due to modelling errors, e.g. in case of domain or electrode geometry mis-modelling or for high contact impedance variations.

To further encourage piecewise constant conductivities, one could add a nonlinear filter (median, total variation, etc.) after line 11. We did not find this necessary in our experiments.

\section{NUMERICAL RESULTS}

Here we demonstrate the performance of the proposed inversion scheme MANTIS, in particular the impact of the weighting schemes $W_{\mathcal{S}}, W_{\sigma}$ and $W_{\mathcal{S}, \sigma}$ in section 4.2 and the impact of the conductivity transformations $t_{\log }$ and $t_{\widehat{\alpha}}$ in section 4.3 .

4.1. Numerical setting. All simulated measurement data are generated using the FEM on very fine triangulations $(\approx 50000$ triangles). To avoid inverse crime, these triangulations are not refinements of the discretizations $\mathcal{P}(\approx 18000$ triangles $)$ used for inversion $[6$. Noisy data

\footnotetext{
${ }^{5}$ FEM triangulations should be refined near the electrodes and should not be too irregular.

${ }^{6}$ We use extremely fine FEM meshes to minimize discretization effects of the forward solver when comparing the results. In practice, far fewer triangles are sufficient.
} 


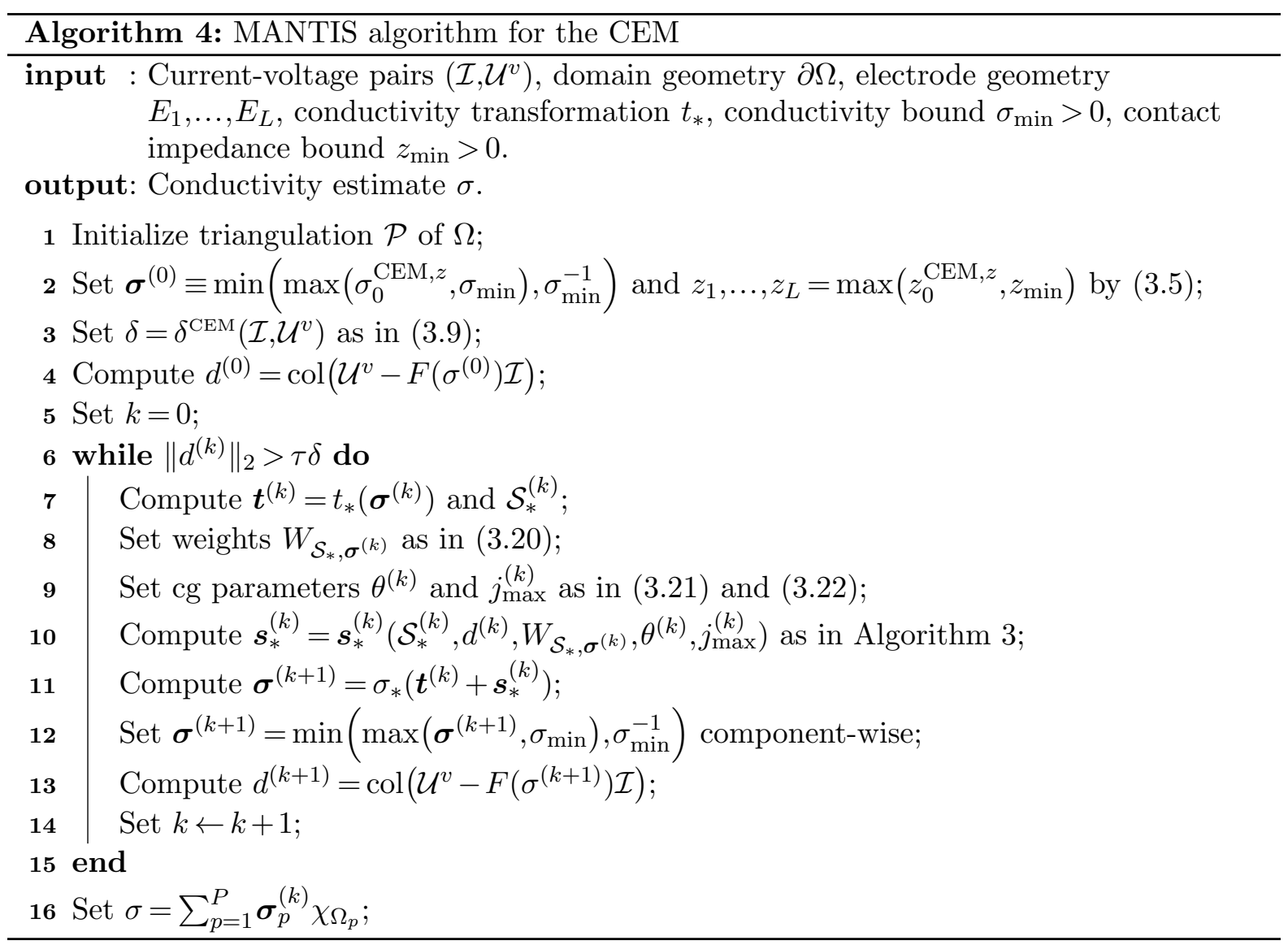

$\mathcal{U}^{v}=\mathcal{U}+N^{v(\eta)}$ is generated as in section 3.2. The relative error between a reconstructed conductivity $\sigma_{\text {rec }}$ and the exact solution $\sigma$ is computed numerically as

$$
\varepsilon=\frac{1}{|\Delta|} \int_{\Delta} \frac{\left|P_{\Delta} \sigma_{\mathrm{rec}}(x)-P_{\Delta} \sigma(x)\right|}{\left|P_{\Delta} \sigma(x)\right|} \mathrm{d} x
$$

where $P_{\Delta}$ is a piecewise constant interpolation on a very fine triangulation $\Delta$ of $\Omega$ with area $|\Delta|$. We denote the total count of Newton and cg iterations by $n_{\mathrm{Nt}}$ and $n_{\mathrm{cg}}$, respectively. To avoid constrainedness in untransformed reconstruction, we approximate $t_{\text {Id }}$ by $t_{\alpha_{\sigma}}$ with $\alpha_{\sigma}=1-10^{-4}$.

Test conductivity. To investigate the capability of reconstructing both low and high contrasts, we consider the test conductivity of Figure 2 with two background conductivities which differ by a factor of 2 and three inclusions which differ by a factor of 100. Conductivity plots are truncated to the interval $\left[0.9 \cdot 10^{-1}, 1.1 \cdot 10^{1}\right]$ in logarithmic scale.

Measurement settings. We assume typical measurement equipment to have 16-64 channels and $\eta \approx 0.1-0.3 \%$ relative error per measurement. To study the impact of the measurement setting, we consider two configurations: Setting A with few electrodes and moderate contact impedances and measurement error, and setting B (gray, in brackets) with more electrodes and lower contact impedances and measurement error. Setting A (B) consists of 16 (64) electrodes covering $50 \%$ of the boundary and contact impedances $z_{l}=1.0 \mathrm{e}-1(2.5 \mathrm{e}-2) \pm 10 \%, l=1, \ldots, L$, as in (3.6). We use a full set of adjacent currents and simulate noisy measurements by (3.11) for $\eta=0.3 \%(0.1 \%)$. The resulting initial guesses for these settings are $z_{0}^{\mathrm{CEM}, z}=9.63 \mathrm{e}-2(2.51 \mathrm{e}-2)$ and $\sigma_{0}^{\mathrm{CEM}, \mathrm{z}}=0.84$ (0.87). The true and estimated errors are $\delta=1.54 \mathrm{e}-2(1.02 \mathrm{e}-2)$ and $\delta^{\text {CEM }}=1.51 \mathrm{e}-2(1.05 \mathrm{e}-2)$, respectively. 


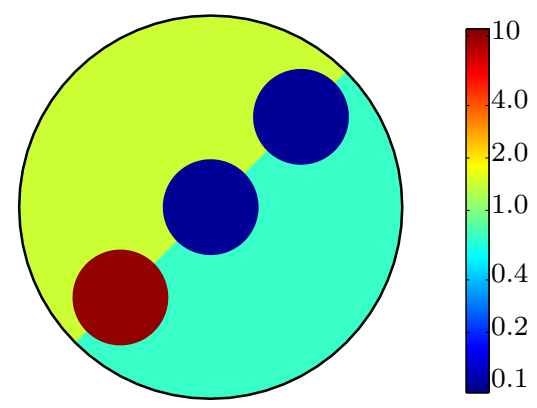

FIGURE 2. High-contrast test conductivity. Background $\sigma=\sqrt{2}$ (top-left, light green) and $\sigma=\frac{1}{\sqrt{2}}$ (bottom-right, turquoise), inclusions $\sigma=10$ (bottom-left, red) and $\sigma=10^{-1}$ (others, blue).

4.2. Impact of the weights. Figure 3 shows reconstructions using MANTIS without conductivity transformation and with $W=\operatorname{Id}(\mathrm{a}), W_{\mathcal{S}}(\mathrm{b}), W_{\boldsymbol{\sigma}}(\mathrm{c})$ and $W_{\mathcal{S}, \boldsymbol{\sigma}}(\mathrm{d})$; see (3.19) and (3.18). Table 5, rows 1-4 and 9-12 list some numerical information. Clearly, ignoring the sensitivities and underlying conductivities leads to slow convergence and highly oscillatory solutions. Consequently, the different background conductivities cannot be distinguished well and the inclusions are not detected reliably. Note that all solutions reach the residual discrepancy $\tau \delta$, but the inversion without weights gets unstable before the discrepancy criterion is met.

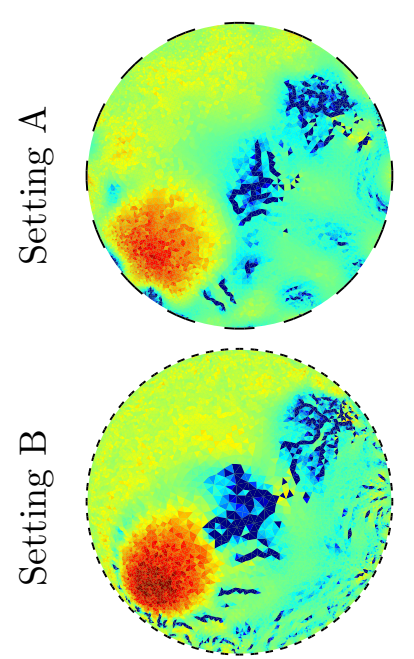

(a) $W=$ Id

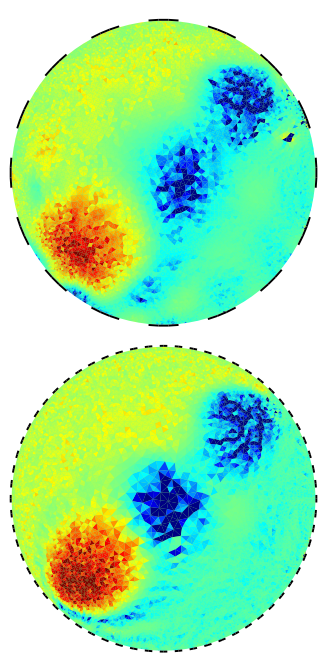

(b) $W_{\sigma}$

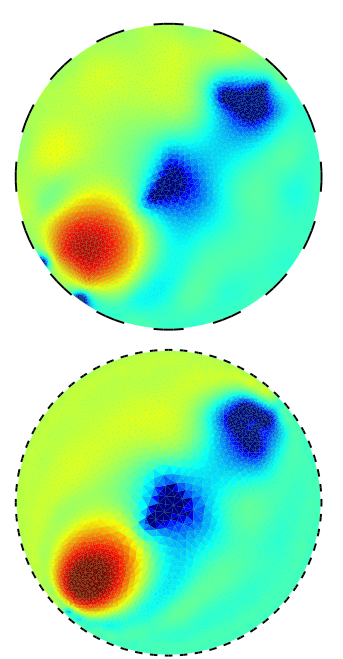

(c) $W_{\mathcal{S}}$

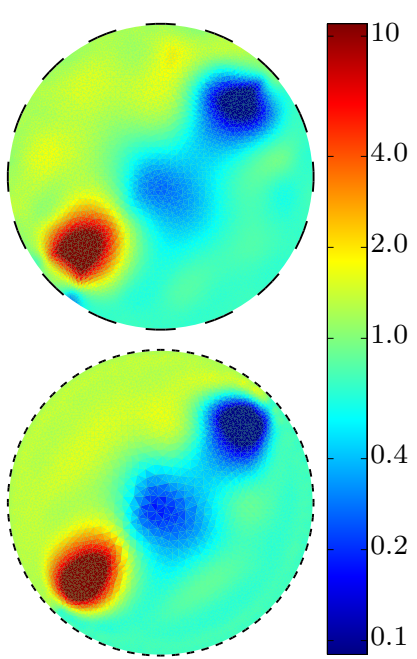

(d) $W_{\mathcal{S}, \boldsymbol{\sigma}}$

FIGURE 3. Reconstructions of the test conductivity for different weighting schemes. The reconstruction prior, implemented by $W_{\mathcal{S}, \sigma}$, encourages piecewise constant conductivities. The small and large contrasts are resolved.

4.3. Impact of the transformation. Figure 4 shows reconstructions using the transformations $t_{\log }$ and $t_{\widehat{\alpha}}$ for weights $W=\mathrm{Id}$ and $W_{\mathcal{S}_{*}, \sigma}$. Table 5 , rows $5-8$ and $13-16$ list the reconstruction properties. With unit weights, the transformations significantly improve the reconstruction speed and accuracy; compare Figure $3 \mathrm{a}$ with $4 \mathrm{a}, 4 \mathrm{~b}$. Using the weights $W_{\mathcal{S}_{*}, \sigma}$, the transformations improve the reconstructions just slightly; compare Figure $3 \mathrm{~d}$ with $4 \mathrm{c}$, $4 \mathrm{~d}$. This is because the reconstruction prior diminishes the effect of the particular transform. We observe that the $\log$ transform results in slightly faster convergence while the $\widehat{\alpha}$ transform results in slightly lower error in our simulations. 


\begin{tabular}{|c|c|c|c|c|c|c|c|}
\hline & weights & transf. & rel. error & $n_{\mathrm{Nt}}$ & $n_{\mathrm{cg}}$ & $\min (\sigma)$ & $\max (\sigma)$ \\
\hline \multirow{8}{*}{ 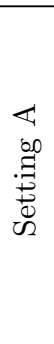 } & Id & $t_{\text {Id }}$ & $74.5 \%$ & 84 & 509 & $1.0 \mathrm{e}-4^{1}$ & $7.3 \mathrm{e}+0$ \\
\hline & $W_{\sigma}$ & $t_{\mathrm{Id}}$ & $60.6 \%$ & 50 & 178 & $1.1 \mathrm{e}-4$ & $1.4 \mathrm{e}+1$ \\
\hline & $W_{\mathcal{S}}$ & $t_{\mathrm{Id}}$ & $49.0 \%$ & 11 & 58 & $3.3 \mathrm{e}-4$ & $7.7 \mathrm{e}+0$ \\
\hline & $W_{\mathcal{S}, \boldsymbol{\sigma}}$ & $t_{\mathrm{Id}}$ & $46.1 \%$ & 10 & 43 & $5.7 \mathrm{e}-2$ & $1.9 \mathrm{e}+1$ \\
\hline & Id & $t_{\log }$ & $56.6 \%$ & 15 & 98 & $4.8 \mathrm{e}-2$ & $2.4 \mathrm{e}+2$ \\
\hline & Id & $t_{\widehat{\alpha}}$ & $59.7 \%$ & 19 & 135 & $1.6 \mathrm{e}-1$ & $1.5 \mathrm{e}+1$ \\
\hline & $W_{\mathcal{S}_{\log }, \boldsymbol{\sigma}}$ & $t_{\log }$ & $46.0 \%$ & 9 & 41 & $6.3 \mathrm{e}-2$ & $2.4 \mathrm{e}+1$ \\
\hline & $W_{\mathcal{S}_{\widehat{\alpha}}, \boldsymbol{\sigma}}$ & $t_{\widehat{\alpha}}$ & $44.9 \%$ & 10 & 45 & $7.0 \mathrm{e}-2$ & $2.1 \mathrm{e}+1$ \\
\hline \multirow{8}{*}{ 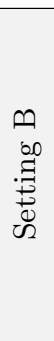 } & Id & $t_{\mathrm{Id}}$ & $64.4 \%$ & 240 & 5329 & $1.0 \mathrm{e}-4^{1}$ & $1.1 \mathrm{e}+1$ \\
\hline & $W_{\boldsymbol{\sigma}}$ & $t_{\mathrm{Id}}$ & $47.2 \%$ & 92 & 1071 & $2.5 \mathrm{e}-4$ & $1.8 \mathrm{e}+1$ \\
\hline & $W_{\mathcal{S}}$ & $t_{\mathrm{Id}}$ & $40.8 \%$ & 18 & 103 & $9.2 \mathrm{e}-4$ & $1.4 \mathrm{e}+1$ \\
\hline & $W_{\mathcal{S}, \boldsymbol{\sigma}}$ & $t_{\mathrm{Id}}$ & $40.4 \%$ & 12 & 57 & $3.3 \mathrm{e}-2$ & $3.5 \mathrm{e}+1$ \\
\hline & Id & $t_{\log }$ & $46.4 \%$ & 27 & 366 & $1.7 \mathrm{e}-2$ & $1.9 \mathrm{e}+2$ \\
\hline & Id & $t_{\widehat{\alpha}}$ & $46.4 \%$ & 86 & 1100 & $8.8 \mathrm{e}-2$ & $1.8 \mathrm{e}+1$ \\
\hline & $W_{\mathcal{S}_{\log }, \boldsymbol{\sigma}}$ & $t_{\log }$ & $41.5 \%$ & 11 & 48 & $3.8 \mathrm{e}-2$ & $4.2 \mathrm{e}+1$ \\
\hline & $W_{\mathcal{S}_{\widehat{\alpha}}, \boldsymbol{\sigma}}$ & $t_{\widehat{\alpha}}$ & $39.8 \%$ & 12 & 59 & $4.1 \mathrm{e}-2$ & $3.4 \mathrm{e}+1$ \\
\hline
\end{tabular}

TABle 5. Relative error, Newton and cg iteration count and minimum and maximum conductivity value for different reconstruction parameters. The reconstruction prior, implemented by $W_{\mathcal{S}, \sigma}$, significantly improves reconstruction speed and quality. Untransformed reconstruction $\left(t_{\mathrm{Id}}\right)$ promotes very small conductivity coefficients.

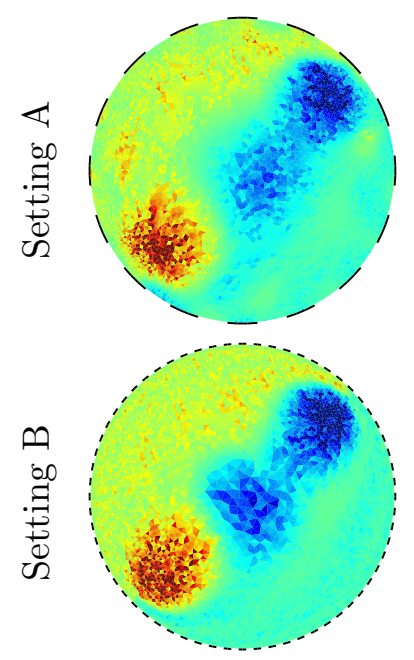

(a) $t_{\log }, W=\mathrm{Id}$

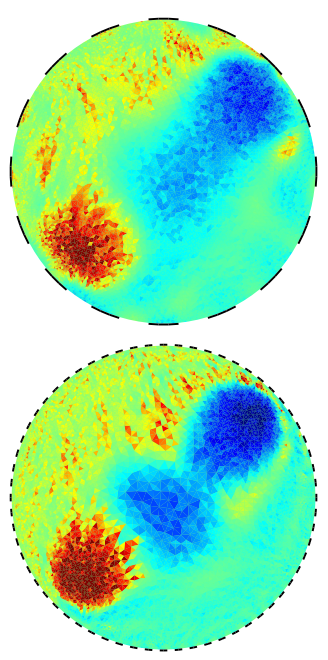

(b) $t_{\widehat{\alpha}}, W=\mathrm{Id}$

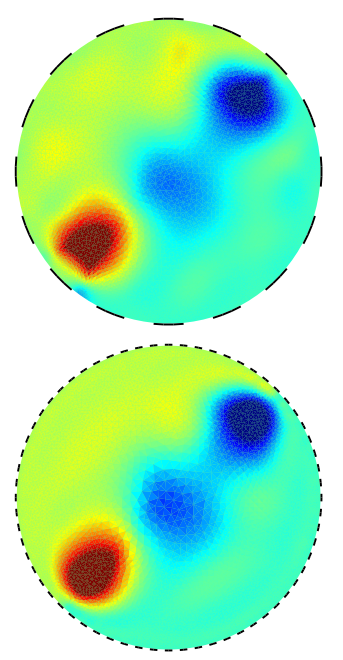

(c) $t_{\log }, W_{\mathcal{S}_{\log }, \boldsymbol{\sigma}}$

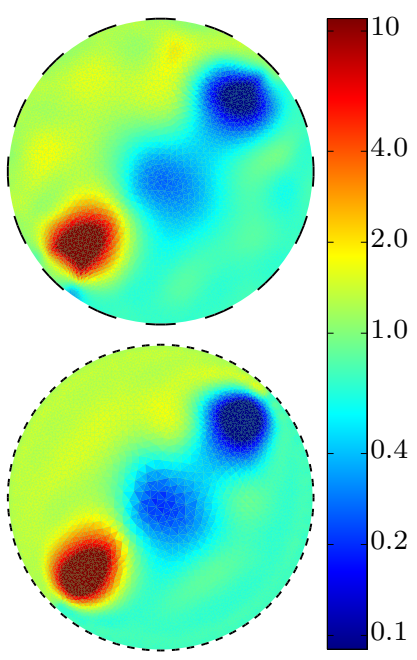

(d) $t_{\widehat{\alpha}}, W_{\mathcal{S}_{\widehat{\alpha}}, \boldsymbol{\sigma}}$

FIGURE 4. Reconstructions of the test conductivity for different transformations with and without weighting.

4.4. Reconstructions with resolution-controlled meshes. In [22, the authors introduced the concept of adaptive, resolution-controlled conductivity discretizations, which improve the speed and accuracy of the inversion. These meshes are designed to satisfy

$$
\left\|F\left(1+\chi_{\Omega_{p}}\right)-F(1)\right\| \approx \text { const. for all } p=1, \ldots, P .
$$

Since

$$
\left\|\mathcal{S}_{p}\right\|_{2}=\left\|F^{\prime}(\sigma)\left[\chi_{\Omega_{p}}\right] \mathcal{I}\right\|_{\text {Fro }}=\left\|F\left(\sigma+\chi_{\Omega_{p}}\right)-F(\sigma)\right\|_{\text {Fro }}+\mathrm{o}\left(\left\|\chi_{\Omega_{p}}\right\|_{\infty}\right),
$$

the regularization mechanism of these meshes is similar to using the weights $W_{\mathcal{S}}$. The reconstructions shown in Figure 5a illustrate how these meshes prevent instability for $t_{\mathrm{Id}}$ and $W=\mathrm{Id}$, 
but have difficulties with high contrast reconstructions as very small conductivity coefficients are promoted. As a consequence, very many Newton iterations are needed to reach the discrepancy criterion and recover the conducting inclusion. When using adaptive meshes and applying the transformation $t_{\widehat{\alpha}}$ and the weights $W_{\mathcal{S}_{\widehat{\alpha}}, \sigma}$, convergence is fast, but no improvement over using the generic discretization $\mathcal{P}$ is achieved (see Table 6). This illustrates the property of the reconstruction prior to diminish the impact of the particular choice of discretization. For that reason, further analysis of the different discretization effects is omitted.

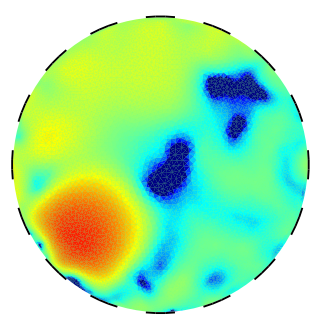

(a) $t_{\text {Id }}, W=$ Id

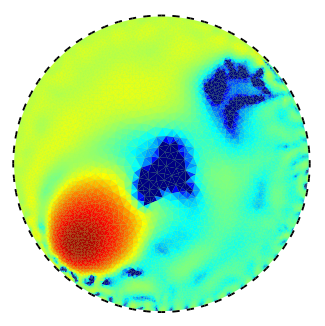

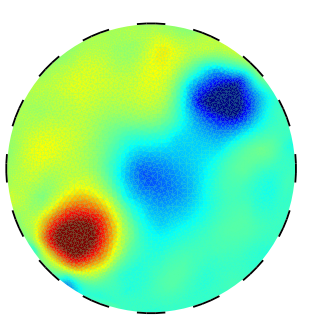

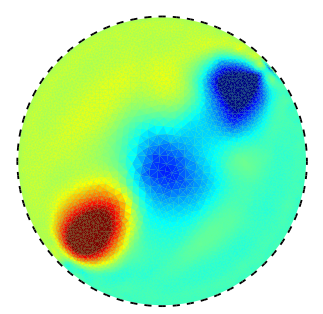

(b) $t_{\widehat{\alpha}}, W_{\mathcal{S}_{\widehat{\alpha}}, \sigma}$

FiguRE 5. Reconstructions on adaptive meshes. The conductivity discretizations are refined dynamically with 108-1470 (139-3687) cells as described in 22, section 5.6]. The instability is resolved and low contrasts are detected, but convergence speed and high contrasts still suffer from the nonlinearity.

\begin{tabular}{rccrrrrr}
\hline & weights & param. & rel. error & $n_{\mathrm{Nt}}$ & $n_{\mathrm{cg}}$ & $\min (\sigma)$ & $\max (\sigma)$ \\
\hline \multirow{2}{*}{$\varangle$} & $\mathrm{Id}$ & $t_{\mathrm{Id}}$ & $61.4 \%$ & 422 & 2097 & $1.0 \mathrm{e}-4^{1}$ & $5.0 \mathrm{e}+0$ \\
& $W_{\mathcal{S}_{\widehat{\alpha}}, \boldsymbol{\sigma}}$ & $t_{\widehat{\alpha}}$ & $45.0 \%$ & 12 & 50 & $6.4 \mathrm{e}-2$ & $1.6 \mathrm{e}+1$ \\
\cline { 2 - 8 } & $\mathrm{Id}$ & $t_{\mathrm{Id}}$ & $48.5 \%$ & 1822 & 16882 & $1.0 \mathrm{e}-4$ & $8.3 \mathrm{e}+0$ \\
& $W_{\mathcal{S}_{\widehat{\alpha}}, \boldsymbol{\sigma}}$ & $t_{\widehat{\alpha}}$ & $41.4 \%$ & 37 & 87 & $3.7 \mathrm{e}-2$ & $2.7 \mathrm{e}+1$ \\
\hline
\end{tabular}

${ }^{1}$ Bounded to $\sigma_{\min }$ during inversion.

TABLE 6. Reconstruction properties when using adaptive meshes.

4.5. Tank data reconstructions. The MANTIS algorithm is particularly tailored for measured data, where the contact impedances, background conductivity and data noise are unknown: There are no free design parameters that need to be chosen manually for the particular setting, and no measured homogeneous calibration data is needed. Figure 6 shows MANTIS reconstructions (using $t_{\widehat{\alpha}}$ and $W_{\mathcal{S}_{\widehat{\alpha}}, \sigma}$ ) of various saline tank experiments with metal (conducting) and plastic (resistive) inclusions. The data were kindly provided by Aku Seppänen (University of Eastern Finland) and Stratos Staboulis (Aalto University). For details, see [8]. The boundary geometry and the positions of the 16 electrodes are estimated from the photographs. The data range of each plot is centered about the estimated background conductivity $\sigma_{0}^{\mathrm{CEM}, z}$ on a logarithmic scale. The estimated parameters and some reconstruction information are shown in Table 7. Note that techniques to recover the electrode locations [6] or the boundary shape [7] can readily be included into MANTIS by computing the Fréchet derivative also with respect to the electrode positions and the boundary parametrization.

\section{Conclusions}

Solving the inverse conductivity problem from measured electrode data poses several difficulties: Some model parameters are unknown, the problem is highly nonlinear, ill-posed and under-determined, the data is corrupted by noise, the problem needs to be discretized for numerical inversion, and there might be inaccuracies when modelling the domain geometry.

Many common approaches, like Tikhonov-regularized inversion, have "abstract" regularization or smoothing parameters which handle multiple issues (level of under-determinedness, illposedness, nonlinearity, mis-modelling, etc.) at once. Consequently, such parameters are highly 


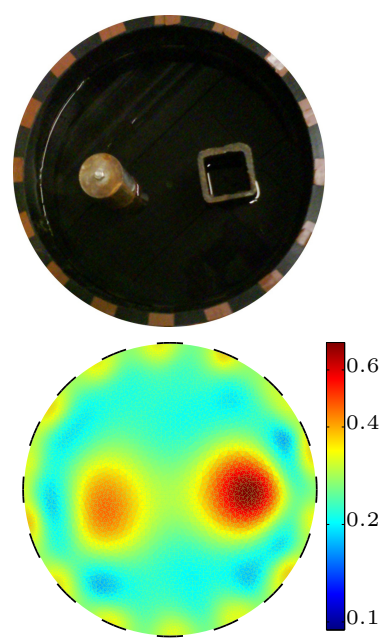

(a)

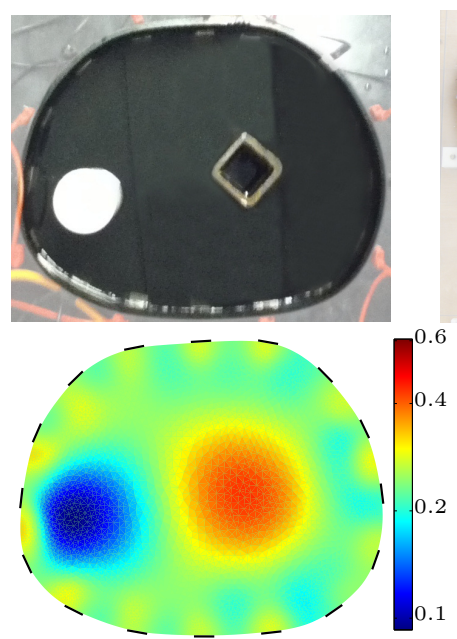

(b)

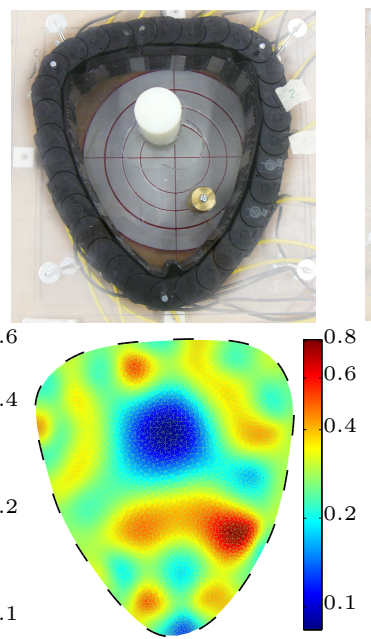

(c)

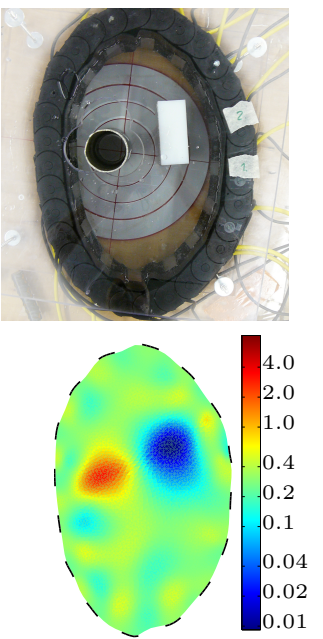

(d)

FiguRE 6. Reconstructions from tank experiments. The domain geometry was estimated from the photographs shown in the top row. The geometry modelling error leads to oscillations of the solutions near the boundary.

\begin{tabular}{ccccrrrr}
\hline Setting & $\sigma_{0}^{\text {CEM }}$ & $z_{0}^{\text {CEM }}$ & $\delta^{\text {CEM }}$ & $n_{\text {Nt }}$ & $n_{\text {cg }}$ & $\min (\sigma)$ & $\max (\sigma)$ \\
\hline (a) & $2.64 \mathrm{e}-1$ & $7.67 \mathrm{e}-1$ & $1.43 \mathrm{e}-2$ & 12 & 55 & $1.8 \mathrm{e}-1$ & $6.6 \mathrm{e}-1$ \\
(b) & $2.38 \mathrm{e}-1$ & $6.14 \mathrm{e}-2$ & $1.98 \mathrm{e}-2$ & 24 & 41 & $1.0 \mathrm{e}-1$ & $4.1 \mathrm{e}-1$ \\
(c) & $2.61 \mathrm{e}-1$ & $1.00 \mathrm{e}-4^{1}$ & $1.55 \mathrm{e}-2$ & 13 & 85 & $9.4 \mathrm{e}-2$ & $7.4 \mathrm{e}-1$ \\
(d) & $2.78 \mathrm{e}-1$ & $1.00 \mathrm{e}-4^{1}$ & $1.89 \mathrm{e}-2$ & 27 & 158 & $1.0 \mathrm{e}-2$ & $3.0 \mathrm{e}-0$ \\
\hline
\end{tabular}

${ }^{1}$ Initialized to $z_{\min }$.

TABLE 7. Reconstruction properties of the tank experiments.

problem-specific and often need to be specified manually over several orders of magnitude to obtain good results.

In this work, we investigated each issue arising in the ICP individually, and presented a set of methods to resolve them without introducing abstract design parameters. The result is an inversion scheme which is simple to implement and applicable to a wide range of settings "out of the box", i.e. without manually choosing or fine-tuning parameters. This was achieved by estimating the contact impedances, background-conductivity and noise level from the measured data, transforming the problem to reduce nonlinearity, resolving the under-determinedness of the linearized problem by a prior on the conductivity, and applying an inexact Newton-type method with Morozov's discrepancy principle to handle the ill-posedness.

The effects of each step were evaluated numerically, and the performance of the scheme was demonstrated for simulated data with high and low conductivity contrasts and for measured tank data.

Note that all considerations extend to three dimensions, so MANTIS can be used also for 3D reconstructions.

\section{Acknowledgements}

The authors thank Aku Seppänen, Stratos Staboulis and Nuutti Hyvönen for providing the data of the tank experiments. 


\section{Appendix A. Noise estimate from the ND ASYMmetry}

Given the exact current-voltage pairs $(\mathcal{I}, \mathcal{U})$ and a noise matrix $N^{v} \in \mathbb{R}^{L \times M}$ with independent identically distributed (i.i.d.) zero mean entries of variance $v>0$, consider the matrix

$$
E^{v}=\left(\mathcal{U}+N^{v}\right) \mathcal{I}^{+}-\left(\left(\mathcal{U}+N^{v}\right) \mathcal{I}^{+}\right)^{\top}=\underbrace{\mathcal{U} \mathcal{I}^{+}-\left(\mathcal{U} \mathcal{I}^{+}\right)^{\top}}_{=0 \text { (symmetry) }}+N^{v} \mathcal{I}^{+}-\left(N^{v} \mathcal{I}^{+}\right)^{\top} .
$$

The matrix has vanishing diagonal entries, thus

$$
E_{k, l}^{v}= \begin{cases}0, & k=l, \\ \sum_{j=1}^{L} N_{k, j}^{v} \mathcal{I}_{j, l}^{+}-N_{l, j}^{v} \mathcal{I}_{j, k}^{+}, & 1 \leq k \neq l \leq L .\end{cases}
$$

In particular, each entry is a sum of i.i.d. zero mean random entries. From the linearity of the expected value, we get

$$
\begin{aligned}
\mathbb{E}\left\|E^{v}\right\|_{\text {Fro }}^{2} & =\sum_{k, l=1}^{L} \mathbb{E}\left(E_{k, l}^{v}\right)^{2}=\sum_{k \neq l} \sum_{j=1}^{L}\left(\mathcal{I}_{j, l}^{+}\right)^{2} \underbrace{\mathbb{E}\left(N_{k, j}^{v}\right)^{2}}_{=v}+\left(\mathcal{I}_{j, k}^{+}\right)^{2} \underbrace{\mathbb{E}\left(N_{l, j}^{v}\right)^{2}}_{=v} \\
& =v \sum_{k \neq l}\left\|\mathcal{I}_{l}^{+}\right\|_{2}^{2}+\left\|\mathcal{I}_{k}^{+}\right\|_{2}^{2}=2(L-1)\left\|\mathcal{I}^{+}\right\|_{\text {Fro }}^{2} v .
\end{aligned}
$$

\section{Appendix B. Proof of theorem 1}

B.1. Linearly dependent case. Let $K, P \in \mathbb{N}, w_{1}, \ldots, w_{P}>0$ and $W=\operatorname{diag}\left(w_{1}, \ldots, w_{P}\right)$. Further, let $X=\mathbb{R}^{P}$ equipped with the inner product $\langle x, y\rangle_{W}=x^{\top} W y$ and $Y=\mathbb{R}^{K}$ equipped with the standard inner product. Consider

$$
\mathcal{S}=\left(\mathcal{S}_{1}, \ldots, \mathcal{S}_{P}\right) \in \mathbb{R}^{K \times P}
$$

as an operator from $X$ to $Y$ and let $N=\operatorname{rank}(\mathcal{S}) \leq \min \{K, P\}$.

Denote, for some $d \in \mathbb{R}^{K}$, by $s^{+W}=s^{+W}(d) \in \mathbb{R}^{P}$ the (unique) minimizer of $\|\mathcal{S} s-d\|_{2}$ in $\mathcal{N}(\mathcal{S})^{\perp_{W}}$, the $W$-orthogonal complement of the Null-space of $\mathcal{S}$. Let $\mathcal{S}=U \Sigma V^{*}$ the (reduced) singular value decomposition of the operator $\mathcal{S}$ with singular values $\sigma_{1}>\ldots>\sigma_{N}>0$ and singular vectors $v^{(1)}, \ldots, v^{(N)} \in X, u^{(1)}, \ldots, u^{(N)} \in Y$, that is

$$
U=\left(u^{(1)}|\ldots| u^{(N)}\right), \Sigma=\operatorname{diag}\left(\sigma_{1}, \ldots, \sigma_{N}\right), V=\left(v^{(1)}|\ldots| v^{(N)}\right),
$$

$U$ unitary in $\left(\operatorname{Range}(\mathcal{S}),\|\cdot\|_{2}\right), V$ unitary in $\left(\mathcal{N}(\mathcal{S})^{\perp_{W}},\|\cdot\|_{W}\right)$, thus $U^{*}=U^{\top}, V^{*}=V^{\top} W$. Then, $\boldsymbol{s}^{+W}$ is given by the pseudo-inverse $\mathcal{S}^{+W}$ of $\mathcal{S}$ as

$$
s^{+W}(d)=\mathcal{S}^{+W} d=\left(V \Sigma^{-1} U^{*}\right) d .
$$

We now want to investigate under which circumstances two coefficients $\boldsymbol{s}_{p}^{+W}(d), \boldsymbol{s}_{q}^{+W}(d)$ are proportional for all $d \in Y$. Denote by $v_{\top}^{(1)}, \ldots, v_{\top}^{(P)} \in \mathbb{R}^{N}$ the columns of $V^{\top}$. For any $\alpha_{p}, \alpha_{q}>0$, we have the equivalence

$$
\begin{aligned}
& \text { B.1) } 0=\alpha_{q} s_{q}^{+W}-\alpha_{p} s_{p}^{+W}=\left(\alpha_{q} e_{q}-\alpha_{p} e_{p}\right)^{\top} \mathcal{S}^{+W} d=\left(\left(\mathcal{S}^{+W}\right)^{\top}\left(\alpha_{q} e_{q}-\alpha_{p} e_{p}\right)\right)^{\top} d \quad \forall d \in Y \\
& \Longleftrightarrow 0=\left(U^{*}\right)^{\top} \Sigma^{-1} V^{\top}\left(\alpha_{q} e_{q}-\alpha_{p} e_{p}\right) \\
& \Longleftrightarrow 0=\underbrace{\left(U^{*}\right)^{\top} \Sigma^{-1}}_{\text {full rank }(=N)}\left(\alpha_{q} v_{\top}^{(q)}-\alpha_{p} v_{\top}^{(p)}\right) \\
& \Longleftrightarrow \alpha_{p} v_{\top}^{(p)}=\alpha_{q} v_{\top}^{(q)} .
\end{aligned}
$$


Assume now that two columns $\mathcal{S}_{p}$ and $\mathcal{S}_{q}$ are linearly dependent, that is $\mathcal{S}_{q}=\beta \mathcal{S}_{p}$ for some $\beta \in \mathbb{R} \backslash\{0\}$. We observe that

$$
\begin{aligned}
& 0=\mathcal{S}_{q}-\beta \mathcal{S}_{p}=\mathcal{S}\left(e_{q}-\beta e_{p}\right)=\left(U \Sigma V^{*}\right)\left(e_{q}-\beta e_{p}\right)=\underbrace{U \Sigma}_{\text {full rank }(=N)} V^{\top} W\left(e_{q}-\beta e_{p}\right) \\
\Longleftrightarrow & 0=V^{\top} W\left(e_{q}-\beta e_{p}\right) \\
\Longleftrightarrow & \beta w_{p} v_{\top}^{(p)}=w_{q} v_{\top}^{(q)} .
\end{aligned}
$$

Equivalence between (B.1) and (B.2) holds for $\frac{\alpha_{p}}{\alpha_{q}}=\frac{\beta w_{p}}{w_{q}}$ in which case $\alpha_{q} s_{q}^{+W}=\alpha_{p} s_{p}^{+W} \Longleftrightarrow$ $\mathcal{S}_{q}=\beta \mathcal{S}_{p}$. Since $|\beta|=\frac{\left\|\mathcal{S}_{q}\right\|_{2}}{\left\|\mathcal{S}_{p}\right\|_{2}}$, we get $\frac{\alpha_{p}}{\alpha_{q}}=\frac{\operatorname{sign}(\beta)\left\|\mathcal{S}_{q}\right\|_{2} w_{p}}{\left\|\mathcal{S}_{p}\right\|_{2} w_{q}}$ which yields Theorem 1 (b); (a) follows for $w_{1, \ldots, P} \equiv 1$.

B.2. Almost linearly dependent case. Now assume that $\mathcal{S}_{p}$ and $\mathcal{S}_{q}$ are almost linearly dependent, that is

$$
\mathcal{S}_{q}=\beta \mathcal{S}_{p}+\kappa \boldsymbol{k} \quad \text { for } \quad \beta \in \mathbb{R} \backslash\{0\}, \mathcal{S}_{p} \perp \boldsymbol{k} \in \mathbb{R}^{P},\|\boldsymbol{k}\|_{2}=1,0<\kappa \ll\left\|\beta \mathcal{S}_{p}\right\| .
$$

Using above representations, we have that

$$
\kappa \boldsymbol{k}=\mathcal{S}_{q}-\beta \mathcal{S}_{p}=\left(U \Sigma V^{*}\right)\left(e_{q}-\beta e_{p}\right)=U \Sigma\left(w_{q} v_{\top}^{(q)}-\beta w_{p} v_{\top}^{(p)}\right) .
$$

For $\alpha_{p}=\frac{w_{p}}{\left\|\mathcal{S}_{p}\right\|_{2}}, \alpha_{q}=\frac{\operatorname{sign}(\beta) w_{q}}{\left\|\mathcal{S}_{q}\right\|_{2}}$ and since $\beta=\operatorname{sign}(\beta) \frac{\sqrt{\left\|\mathcal{S}_{q}\right\|_{2}^{2}-\kappa^{2}}}{\left\|\mathcal{S}_{p}\right\|_{2}}$ here (Pythagoras), it follows that

$$
\left\|S_{q}\right\|_{2}\left\|\alpha_{q} v_{\top}^{(q)}-\frac{\sqrt{\left\|S_{q}\right\|_{2}^{2}-\kappa^{2}}}{\left\|S_{q}\right\|_{2}} \alpha_{p} v_{\top}^{(p)}\right\|_{2}=\kappa\left\|\Sigma^{-1} U \boldsymbol{k}\right\|_{2} \leq \kappa\left\|\Sigma^{-1}\right\|_{2} .
$$

Then (using the triangle-inequality),

$$
\left\|\alpha_{q} v_{\top}^{(q)}-\alpha_{p} v_{\top}^{(p)}\right\|_{2} \leq \kappa\left\|\Sigma^{-1}\right\|_{2}\left\|\mathcal{S}_{q}\right\|_{2}^{-1}+c_{\kappa}, \quad \text { where } \quad c_{\kappa}=\left|1-\frac{\sqrt{\left\|S_{q}\right\|_{2}^{2}-\kappa^{2}}}{\left\|S_{q}\right\|_{2}}\right|\left|\alpha_{p}\right|\left\|v_{\top}^{(p)}\right\|_{2},
$$

thus $c_{\kappa}=\mathcal{O}\left(\kappa^{2}\right)$ as $\kappa \rightarrow 0$. Again using the pseudo-inverse representations of the solution coefficients $\boldsymbol{s}_{p}^{+W}, \boldsymbol{s}_{q}^{+W}$, we get

$$
\begin{aligned}
\left|\alpha_{q} s_{q}^{+W}-\alpha_{p} s_{p}^{+W}\right| & =\left|\left(\left(U^{*}\right)^{\top} \Sigma^{-1} V^{\top}\left(\alpha_{q} e_{q}-\alpha_{p} e_{p}\right)\right)^{\top} d\right| \\
& \leq\left\|\Sigma^{-1}\right\|_{2}\left\|\alpha_{q} v_{\top}^{(q)}-\alpha_{p} v_{\top}^{(p)}\right\|_{2}\|d\|_{2} \leq\left\|\Sigma^{-1}\right\|_{2}\left(\kappa\left\|\Sigma^{-1}\right\|_{2}\left\|\mathcal{S}_{q}\right\|_{2}^{-1}+c_{\kappa}\right)\|d\|_{2} .
\end{aligned}
$$

Note that in this expression, the singular values of $\mathcal{S}$ and thus $\Sigma^{-1}$ depend on $\boldsymbol{k}$ and $\kappa$. Recalling that $\boldsymbol{k}=\frac{1}{\kappa}\left(\mathcal{S}_{q}-\beta \mathcal{S}_{p}\right) \in \operatorname{span}\left\{\mathcal{S}_{1}, \ldots, \mathcal{S}_{P}\right\}$ and denoting by $\mathcal{S}^{0}:=\left(\mathcal{S}_{1}, \ldots, \mathcal{S}_{p}, \ldots \mathcal{S}_{q-1}, \beta \mathcal{S}_{p}, \mathcal{S}_{q+1}, \ldots, \mathcal{S}_{P}\right)$ the sensitivity matrix for $\kappa=0$, we have to consider two cases as $\kappa \rightarrow 0$ :

(i) $\operatorname{rank}\left(\mathcal{S}^{0}\right)<\operatorname{rank}(\mathcal{S})$ :

This occurs if and only if $\boldsymbol{k} \notin \operatorname{span}\left\{\mathcal{S}_{1}^{0}, \ldots, \mathcal{S}_{P}^{0}\right\}=\operatorname{span}\left\{\mathcal{S}_{l}: l \in\{1, \ldots, P\} \backslash q\right\}$. From the continuity of the singular values in the matrix entries, we have that the minimum singular value $\sigma_{\min }$ of $\mathcal{S}$ vanishes as $\kappa \rightarrow 0$, thus $\left\|\Sigma^{-1}\right\|_{2}=\sigma_{\min }^{-1} \rightarrow \infty$.

(ii) $\operatorname{rank}\left(\mathcal{S}^{0}\right)=\operatorname{rank}(\mathcal{S})$ :

Again by the continuity of the singular values, $\sigma_{\min }$ approaches the smallest non-zero singular value of $\mathcal{S}^{0}$ as $\kappa \rightarrow 0$, thus $\left\|\Sigma^{-1}\right\|_{2}$ is bounded and $\alpha_{q} s_{q}^{+W} \rightarrow \alpha_{p} s_{p}^{+W}$ as $\kappa \rightarrow 0$.

For $\alpha_{p, q}$ as above, this proves Corollary 3 . 


\section{REFERENCES}

1. K Astala and L Päivärinta, Calderón's inverse conductivity problem in the plane, Annals of Mathematics (2006), 265-299.

2. DC Barber and AD Seagar, Fast reconstruction of resistance images, Clinical Physics and Physiological Measurement 8 (1987), no. 4A, 47.

3. L Borcea, V Druskin, and F Guevara Vasquez, Electrical impedance tomography with resistor networks, Inverse Problems 24 (2008), no. 3, 035013.

4. M Cheney, D Isaacson, JC Newell, S Simske, and JC Goble, NOSER: An algorithm for solving the inverse conductivity problem, International Journal of Imaging Systems and Technology 2 (1990), no. 2, 66-75.

5. MT Clay and TC Ferree, Weighted regularization in electrical impedance tomography with applications to acute cerebral stroke, Medical Imaging, IEEE Transactions on 21 (2002), no. 6, 629-637.

6. J Dardé, H Hakula, N Hyvönen, S Staboulis, and E Somersalo, Fine-tuning electrode information in electrical impedance tomography, Inverse Problems and Imaging 6 (2012), no. 3, 399-421.

7. J Dardé, N Hyvönen, A Seppänen, and S Staboulis, Simultaneous reconstruction of outer boundary shape and admittivity distribution in electrical impedance tomography, SIAM Journal on Imaging Sciences 6 (2013), no. 1, 176-198.

8. _ Simultaneous recovery of admittivity and body shape in electrical impedance tomography: an experimental evaluation, Inverse Problems 29 (2013), no. 8, 085004.

9. B Grychtol and A Adler, Choice of reconstructed tissue properties affects interpretation of lung EIT images, Physiological measurement 35 (2014), no. 6, 1035.

10. DS Holder, Electrical impedance tomography: Methods, history and applications, Series in Medical Physics and Biomedical Engineering, Taylor \& Francis, 2004.

11. JP Kaipio, V Kolehmainen, E Somersalo, and M Vauhkonen, Statistical inversion and monte carlo sampling methods in electrical impedance tomography, Inverse Problems 16 (2000), no. 5, 1487.

12. A Lechleiter and A Rieder, Newton regularizations for impedance tomography: a numerical study, Inverse Problems 22 (2006), no. 6, 1967-1987.

13. HR MacMillan, TA Manteuffel, and SF McCormick, First-order system least squares and electrical impedance tomography, SIAM journal on numerical analysis 42 (2004), no. 2, 461-483.

14. Sungho Oh, Compensation of shape change artifacts and spatially-variant image reconstruction problems in electrical impedance tomography, Ph.D. thesis, University of Florida, 2009.

15. W Polydorides, N Lionheart, A MATLAB-based toolkit for three-dimensional electrical impedance tomography: A contribution to the eidors project, Measurement Science and Technology 13 (2002), no. 12.

16. A Rieder, On the regularization of nonlinear ill-posed problems via inexact Newton iterations, Inverse Problems 15 (1999), no. 1, 309-327.

17. __ Inexact Newton regularization using conjugate gradients as inner iteration, SIAM Journal on $\mathrm{Nu}-$ merical Analysis 43 (2005), no. 2, 604-622.

18. RJ Sadleir, SU Zhang, AS Tucker, and Sungho Oh, Imaging and quantification of anomaly volume using an eight-electrode 'hemiarray' EIT reconstruction method, Physiological measurement 29 (2008), no. 8, 913-927.

19. E Somersalo, M Cheney, and D Isaacson, Existence and uniqueness for electrode models for electric current computed tomography, SIAM Journal on Applied Mathematics 52 (1992), no. 4, 1023-1040.

20. M Vauhkonen, Electrical impedance tomography and prior information, Ph.D. thesis, University of Kuopio, 1997.

21. T Vilhunen, JP Kaipio, PJ Vauhkonen, T Savolainen, and M Vauhkonen, Simultaneous reconstruction of electrode contact impedances and internal electrical properties: I. theory, Measurement Science and Technology 13 (2002), no. 12, 1848-1854.

22. R Winkler and A Rieder, Resolution-controlled conductivity discretization in electrical impedance tomography, SIAM Journal on Imaging Sciences 7 (2014), no. 4, 2048-2077.

Institute for Applied and Numerical Mathematics,

Karlsruhe Institute of Technology, DE-76049 Karlsruhe, Germany. 


\section{IWRMM-Preprints seit 2012}

Nr. 12/01 Branimir Anic, Christopher A. Beattie, Serkan Gugercin, Athanasios C. Antoulas: Interpolatory Weighted-H2 Model Reduction

Nr. 12/02 Christian Wieners, Jiping Xin: Boundary Element Approximation for Maxwell's Eigenvalue Problem

Nr. 12/03 Thomas Schuster, Andreas Rieder, Frank Schöpfer: The Approximate Inverse in Action IV: Semi-Discrete Equations in a Banach Space Setting

Nr. 12/04 Markus Bürg: Convergence of an hp-Adaptive Finite Element Strategy for Maxwell's Equations

Nr. 12/05 David Cohen, Stig Larsson, Magdalena Sigg: A Trigonometric Method for the Linear Stochastic Wave Equation

Nr. 12/06 Tim Kreutzmann, Andreas Rieder: Geometric Reconstruction in Bioluminescence Tomography

Nr. 12/07 Tobias Jahnke, Michael Kreim: Error bound for piecewise deterministic processes modeling stochastic reaction systems

Nr. 12/08 Haojun Li, Kirankumar Hiremath, Andreas Rieder, Wolfgang Freude: Adaptive Wavelet Collocation Method for Simulation of Time Dependent Maxwell's Equations

Nr. 12/09 Andreas Arnold, Tobias Jahnke: On the approximation of high-dimensional differential equations in the hierarchical Tucker format

Nr. 12/10 Mike A. Botchev, Volker Grimm, Marlis Hochbruck: Residual, Restarting and Richardson Iteration for the Matrix Exponential

Nr. 13/01 Willy Dörfler, Stefan Findeisen: Numerical Optimization of a Waveguide Transition Using Finite Element Beam Propagation

Nr. 13/02 Fabio Margotti, Andreas Rieder, Antonio Leitao: A Kaczmarz Version of the ReginnLandweber Iteration for Ill-Posed Problems in Banach Spaces

Nr. 13/03 Andreas Kirsch, Andreas Rieder: On the Linearization of Operators Related to the Full Waveform Inversion in Seismology

Nr. 14/01 Robert Winkler, Andreas Rieder: Resolution-Controlled Conductivity Discretization in Electrical Impedance Tomography

Nr. 14/02 Andreas Kirsch, Andreas Rieder: Seismic Tomography is Locally Ill-Posed

Nr. 14/03 Fabio Margotti, Andreas Rieder: An Inexact Newton Regularization in Banach Spaces based on the Nonstationary Iterated Tikhonov Method

Nr. 14/04 Robert Winkler, Andreas Rieder: Model-Aware Newton-Type Inversion Scheme for Electrical Impedance Tomography

Eine aktuelle Liste aller IWRMM-Preprints finden Sie auf: 


\section{Kontakt}

Karlsruher Institut für Technologie (KIT) Institut für Wissenschaftliches Rechnen und Mathematische Modellbildung

Prof. Dr. Christian Wieners

Geschäftsführender Direktor

Campus Süd

Engesserstr. 6

76131 Karlsruhe

E-Mail:Bettina.Haindl@kit.edu

www. math. kit.edu/iwrmm/

\section{Herausgeber}

Karlsruher Institut für Technologie (KIT) Kaiserstraße 12 | 76131 Karlsruhe

Dezember 2014 